\title{
Cholinergic Coordination of Presynaptic and Postsynaptic Activity Induces Timing-Dependent Hippocampal Synaptic Plasticity
}

\author{
Zhenglin Gu, Patricia W. Lamb, and Jerrel L. Yakel \\ Laboratory of Neurobiology, National Institute of Environmental Health Sciences, National Institutes of Health, Department of Health and Human Services, \\ Research Triangle Park, North Carolina 27709
}

Correlated presynaptic and postsynaptic activity is the key factor in inducing Hebbian plasticity and memory. However, little is known about the physiological events that could mediate such coordination. Correlated cholinergic input induces spike timing-dependent plasticity-like hippocampal synaptic plasticity. Cholinergic receptors are localized to both presynaptic and postsynaptic glutamatergic sites and thus have the potential to coordinate presynaptic and postsynaptic activity to induce plasticity. By directly monitoring presynaptic and postsynaptic activities with genetically encoded calcium indicators in mouse septohippocampal cocultures, we found interactive but independent presynaptic and postsynaptic modulations in the cholinergic-dependent synaptic plasticity. Neither presynaptic nor postsynaptic modulation alone is sufficient, but instead a coordinated modulation at both sites is required to induce the plasticity. Therefore, we propose that correlated cholinergic input can coordinate presynaptic and postsynaptic activities to induce timingdependent synaptic plasticity, providing a novel mechanism by which neuromodulators precisely modulate network activity and plasticity with high efficiency and temporal precision.

\section{Introduction}

Synaptic plasticity has been widely studied and deemed to be a cellular mechanism underlying learning and memory (Bliss and Collingridge, 1993; Martin et al., 2000; Dan and Poo, 2006; Neves et al., 2008). Recent studies have revealed that synaptic plasticity can be greatly facilitated when presynaptic and postsynaptic activities are precisely coordinated, as evidenced in spike timing-dependent plasticity (STDP) (Bell et al., 1997; Magee and Johnston, 1997; Markram et al., 1997; Zhang et al., 1998; Egger et al., 1999; Feldman, 2000), and thus provides a potential mechanism to induce plasticity in a physiological context as a part (or the result) of continuous neural activities. Understanding the mechanisms that could coordinate presynaptic and postsynaptic activities is critical not only to our understanding of synaptic plasticity, but also to the nature of information processing and integration in general underlying higher brain functions.

\footnotetext{
Received May 2, 2012; revised July 6, 2012; accepted July 20, 2012.

Author contributions:Z.G. and J.L.Y. designed research;Z.G. performed research;P.W.L. contributed unpublished reagents/analytic tools; Z.G. analyzed data; Z.G. and J.L.Y. wrote the paper.

This work was supported by the Intramural Research Program of the NIH, National Institute of Environmental Health Sciences. We thank Drs. Charles Romeo, Negin Martin, and Bernd Gloss for virus packaging; Charles J. Tucker and Agnus Janoshazi for assistance with fluorescent microscopy; and Drs. Serena Dudek and Christian Erxleben for comments on the manuscript. We also thank Dr. Karl Deisseroth at Stanford University for ChR2 and synapsin promoter-driven AAV vectors, Dr. Loren Looger at Janelia Farm Research Campus for GCaMP3, Dr. Robert Campbell at University of Alberta for R-GEC01, and Dr. James Wilson at University of Pennsylvania for AAV serotype 9 helper plasmid.

The authors declare no competing financial interests.

Correspondence should be addressed to Jerrel L. Yakel, P.0. Box 12233, Mail Drop F2-08, Research Triangle Park, NC27709. E-mail:yakel@niehs.nih.gov.

DOI:10.1523/JNEUROSCI.2129-12.2012

Copyright $\odot 2012$ the authors $\quad 0270-6474 / 12 / 3212337-12 \$ 15.00 / 0$
}

Acetylcholine (ACh) (Jerusalinsky et al., 1997; Power et al., 2003; Dani and Bertrand, 2007; Kenney and Gould, 2008), as well as other modulatory neurotransmitters (Bailey et al., 2000; Reis et al., 2009), has long been suggested to be heavily involved in synaptic plasticity and various higher brain functions. ACh receptors are distributed to both presynaptic and postsynaptic sites of glutamatergic and GABAergic synapses (Levey et al., 1991; FabianFine et al., 2001; Volpicelli and Levey, 2004; Dani and Bertrand, 2007; Drever et al., 2011), providing the potential capability of coordinating presynaptic and postsynaptic activities. Recent studies have suggested the importance of the timing of applied ACh in modulating high-frequency stimulation (HFS)-induced hippocampal synaptic plasticity (Ji et al., 2001; Ge and Dani, 2005). Moreover, we have recently demonstrated that the activation of endogenous cholinergic inputs can also induce timingdependent synaptic plasticity in the hippocampus, with a temporal precision of milliseconds ( $\mathrm{Gu}$ and Yakel, 2011); this provides an ideal model to study information integration and plasticity induction that involves neuromodulators. In the meantime, newly developed genetically encoded calcium indicators (GECIs) (Tian et al., 2012) have provided the ability to directly monitor neuronal activities at either the synapse or the network level. Differently colored (Tian et al., 2009; Zhao et al., 2011) GECIs have provided excellent tools to monitor presynaptic and postsynaptic components at the same time, greatly facilitating our understanding of the coordinated activities that mediate synaptic plasticity and other neuronal functions.

In this study, we have used a septohippocampal coculture system (Gähwiler and Hefti, 1984; Gähwiler and Brown, 1985; Rimvall et al., 1985; Fischer et al., 1999) instead of acute hip- 
pocampal slices that were used previously for two reasons: first, the ease in expressing GECIs to restricted hippocampal subregions for presynaptic and postsynaptic activity observation, and second, to be able to express the $\alpha 7$ nicotinic ACh receptor (nAChR) subtype to either presynaptic or postsynaptic sites (or both) in $\alpha 7 \mathrm{nAChR}$ knock-out (KO) slices. The latter has been our main tool to dissect out the roles of presynaptic and postsynaptic $\alpha 7 \mathrm{nAChRs}$ in inducing the $\alpha 7$ nAChR-dependent long-term potentiation (LTP) and short-term depression (STD), which has helped us reveal the individual contribution of presynaptic and postsynaptic modulation in synaptic plasticity formation.

\section{Materials and Methods}

Animals and chemicals. $\alpha 7$ nAChR knock-out mice and choline acetyltransferase (ChAT)-Cre transgenic mice (of either sex) were originally purchased from Jackson Laboratory and bred at the National Institute of Environmental Health Sciences (NIEHS). Mice were tattooed and genotyped at day 4 and used for slice culture from days 8 to 12 . All procedures were approved and performed in compliance with NIEHS/NIH Humane Care and Use of Animals in Research protocols. Unless otherwise indicated, general chemicals were obtained from Sigma, and culture media were from Sigma or Invitrogen.

Coculture slice preparation. Slice cultures were prepared as described by Bastrikova et al. (2008), which was adapted from Stoppini et al. (1991). Brain slices of $300 \mu \mathrm{m}$ were cut with a vibratome (Leica, VT1000S). The detachable parts of the vibratome and surgery instruments for dissecting brains were all autoclaved. Briefly, mice (8-12 days old) were anesthetized with isoflurane and decapitated. Brains were quickly removed into ice-cold cutting medium (MEM supplemented with $25 \mathrm{~mm}$ HEPES, $10 \mathrm{~mm}$ Tris-base, $10 \mathrm{~mm}$ glucose, and $3 \mathrm{~mm} \mathrm{MgCl}$, $\mathrm{pH}$ 7.2). Horizontal hippocampal slices and coronal septum slices were cut in cutting medium. The hippocampus and medial septum tissues were then dissected out from the slices and placed next to each other onto the Transwell membrane inserts (Corning) that were prefilled with 1.2 $\mathrm{ml}$ of culture medium, which was prepared as a 2:1 mixture of basal medium Eagle (Sigma) and Earle's balanced salts solution (Sigma) and supplemented with (in mM) $20 \mathrm{NaCl}, 5 \mathrm{NaHCO} 3,0.2 \mathrm{CaCl} 2,1.7 \mathrm{MgSO}_{4}$ 48 glucose, $26.7 \mathrm{HEPES}, 10 \mathrm{ml} / \mathrm{L}$ penicillin-streptomycin (Invitrogen), insulin $(1.32 \mathrm{mg} / \mathrm{L}$ ) (Sigma), and $5 \%$ horse serum (Invitrogen) with $\mathrm{pH}$ 7.2. The slices were stored in a $\mathrm{CO}_{2}$ incubator at $34^{\circ} \mathrm{C}$ and fed twice a week with a half change of media. For the $\alpha 7$ nAChR knock-out cocultures, the septal tissue that provides the cholinergic inputs was from wild-type littermates, and only the hippocampi were from $\alpha 7 \mathrm{nAChR}$ knock-out mice.

Virus infection. All of the plasmids were subcloned to an adenoassociated virus (AAV) vector with a synapsin promoter for neuronal expression. All of the viruses were packaged with serotype 9 helper at the virus core facility at NIEHS. The AAV vector with synapsin promoter (Addgene plasmid no. 26972) and ChR2 (Addgene plasmid no. 20297) plasmids were from Karl Deisseroth at Stanford University (Stanford, CA). The rat $\alpha 7 \mathrm{nAChR}$ plasmid was obtained from James Patrick at the Baylor College of Medicine (Houston, TX) (Séguéla et al., 1993). GCaMP3 (Addgene plasmid no. 22692) was from Loren Looger at Janelia Farm Research Campus (Ashburn, VA). R-GECO1 (Addgene plasmid no. 32444) was from Robert Campbell at the University of Alberta (Edmonton, Alberta, Canada). AAV serotype 9 helper plasmid was from James Wilson at University of Pennsylvania (Philadelphia, PA). The day after culturing, viruses $(5 \mathrm{nl})$ were microinjected to the desired areas with a Drummond "Nanoject" (Drummond Scientific). Experiments were done 7 days after virus infection to allow protein expression and cholinergic innervation into the hippocampus.

Pairing two input pathways by electrical stimulation and an optogenetic approach. Cocultured slices were cut out with the attached Transwell membrane from the inserts and put into a submerged chamber continuously perfused with $95 \% \mathrm{O}_{2} / 5 \% \mathrm{CO}_{2}$ balanced ACSF (in mM: $122 \mathrm{NaCl}$, $2.5 \mathrm{KCl}, 1.3 \mathrm{MgCl}_{2}, 2 \mathrm{CaCl}_{2}, 1.2 \mathrm{NaH}_{2} \mathrm{PO}_{4}, 25 \mathrm{NaHCO}_{3}, 25$ glucose) at a rate of $2 \mathrm{ml} / \mathrm{min}$. Whole-cell patch clamp was performed under guidance of infrared differential interference contrast optics using an Axopatch 200B patch amplifier (Molecular Devices) with a glass pipette filled with an internal solution containing (in $\mathrm{mM}$ ) 120 potassium gluconate, 2 $\mathrm{NaCl}, 5 \mathrm{MgATP}, 0.3 \mathrm{Na}_{2} \mathrm{GTP}, 20 \mathrm{KCl}, 10$ HEPES, 1 EGTA, and 11.3 D-glucose with $\mathrm{pH} \sim 7.2-7.3$ and osmolarity of $\sim 270-280 \mathrm{mOsm}$. Data were digitized with Digidata $1322 \mathrm{~A}$, collected with Clampex, and analyzed with Clampfit. Excitatory postsynaptic currents (EPSCs) were recorded under voltage-clamp at $-60 \mathrm{mV}$.

Evoked EPSCs were recorded from hippocampal CA1 pyramidal neurons by electrically stimulating the Schaffer collateral (SC) pathway. The stimulation intensity was adjusted to evoke a postsynaptic current of about 50-100 pA in amplitude, and the intensity was usually around $20-100 \mu \mathrm{A}$ for $0.1 \mathrm{~ms}$. Light-sensitive ChR2 was selectively expressed in cholinergic neurons by microinjecting a Creinducible AAV containing a double-floxed inverted ChR2 (fused with mCherry for visualization) to the septal tissue from ChAT-Cre transgenic mice. Extensive cholinergic innervations to the hippocampus can be observed 7 days later. Cholinergic terminals expressing ChR2 and mCherry were visualized with $543 \mathrm{~nm}$ light and activated in the stratum oriens $(\mathrm{SO})$ layer above the CA1 pyramidal neuron under patch-clamp recording with $488 \mathrm{~nm}$ light $(20 \mathrm{~ms})$ generated by the Zeiss LSM 510 NLO META system. The exposure time, intensity, and area were controlled by the LSM 510 system. The intensity of the light used to activate ChR2 in processes was usually $7.5 \mathrm{~mW}$ for $20 \mathrm{~ms}$. The cholinergic response (nAChR-mediated currents in CA1 neurons) was usually induced at around $20 \mathrm{~ms}$ after initiating the light exposure (Gu and Yakel, 2011). To achieve a $100 \mathrm{~ms}$ interval for cholinergic inputs before SC inputs, the light exposure was set at $120 \mathrm{~ms}$ before SC input in Clampex. To achieve a $10 \mathrm{~ms}$ interval for cholinergic inputs after SC inputs, the light exposure was set at $10 \mathrm{~ms}$ before SC input to take into account the $20 \mathrm{~ms}$ delay of cholinergic activation by light exposure.

Calcium imaging. Cocultured slices with the attached membrane were cut out and put into a chamber that was continuously perfused with $95 \% \mathrm{O}_{2} / 5 \% \mathrm{CO}_{2}$ balanced ACSF. Calcium imaging was done with a customized spinning disc confocal microscopy. The spinning disk (PerkinElmer) was installed onto an Olympus microscope with a $60 \times$ objective lens (numerical aperture, 0.9). Lasers (Melles Griot) of 488 and $568 \mathrm{~nm}$ were used to excite GCaMP3 and R-GECO1, respectively. Emissions were filtered through bandpass filters 500-550 and 585-630 nm, respectively. The images were collected at $5 \mathrm{~Hz}$ with an iXon3 EMCCD camera (Andor) controlled by Andor iQ imaging software, which was connected with a Sutter Lambda 10 (Sutter Instrument) to select between different channels, a Neos Acousto-optic tunable filter to adjust the laser intensity, and a kinetic trigger device (Andor) to control the image timing. Images were analyzed with NIH ImageJ.

Choline-induced $\alpha 7 n A C h R$ currents in cultured hippocampal slices. $\alpha 7$ nAChR-mediated currents were recorded with whole-cell patch clamp and induced by pressure applications $(50 \mathrm{~ms}, 10 \mathrm{psi})$ of $10 \mathrm{~mm}$ choline (Fayuk and Yakel, 2004) at a 2 min interval through a glass pipette located in the stratum radiatum area (about $150 \mu \mathrm{m}$ away from the soma of the neurons under recording). Pressure was applied through a PPM-2 pneumatic pump (Harvard Apparatus). Tetrodotoxin (1 $\mu \mathrm{m})$ was added to the perfusion ACSF and choline solution.

Statistics. For whole-cell recordings, the amplitude of SC-EPSCs were analyzed with Clampfit. The percent changes were calculated by comparing with the average of $10 \mathrm{~min}$ baseline recording. For calcium imaging, the averages of $1 \mathrm{~s}$ of baseline sampling (i.e., 5 time points) before SC stimulation was used to calculate the percent change of the SC-induced peak calcium intensity. Values were presented as mean \pm SEM. Twotailed Student's $t$ tests were performed to compare changes with the baseline or control.

\section{Results}

Cholinergic inputs induce timing-dependent hippocampal plasticity in septohippocampal cocultures as in acute hippocampal slices

Previous studies have shown that the septohippocampal coculture system provides an excellent model to study cholinergic modulation 

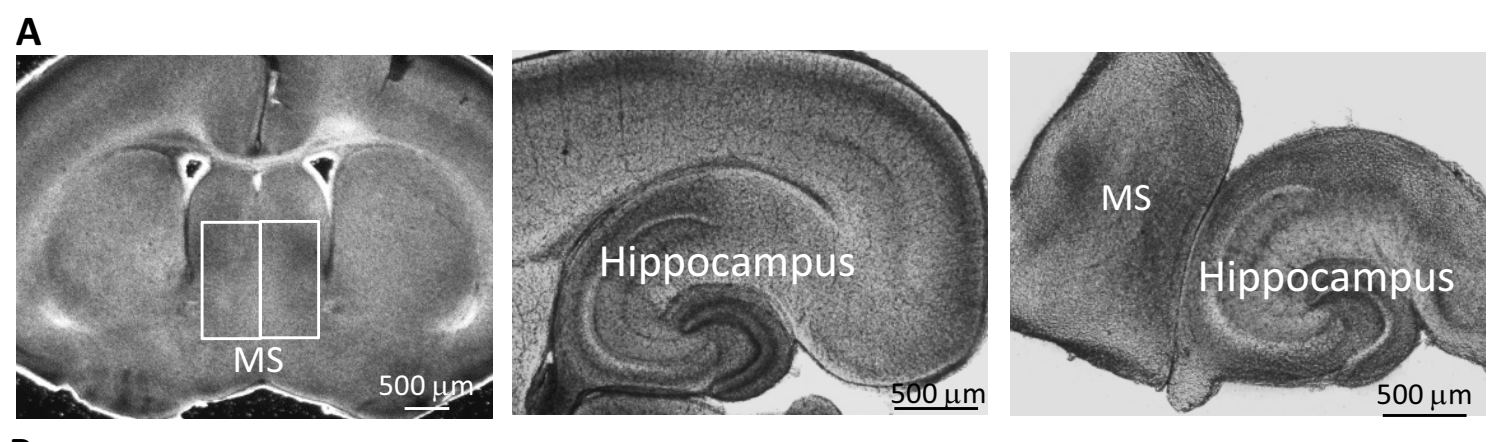

B
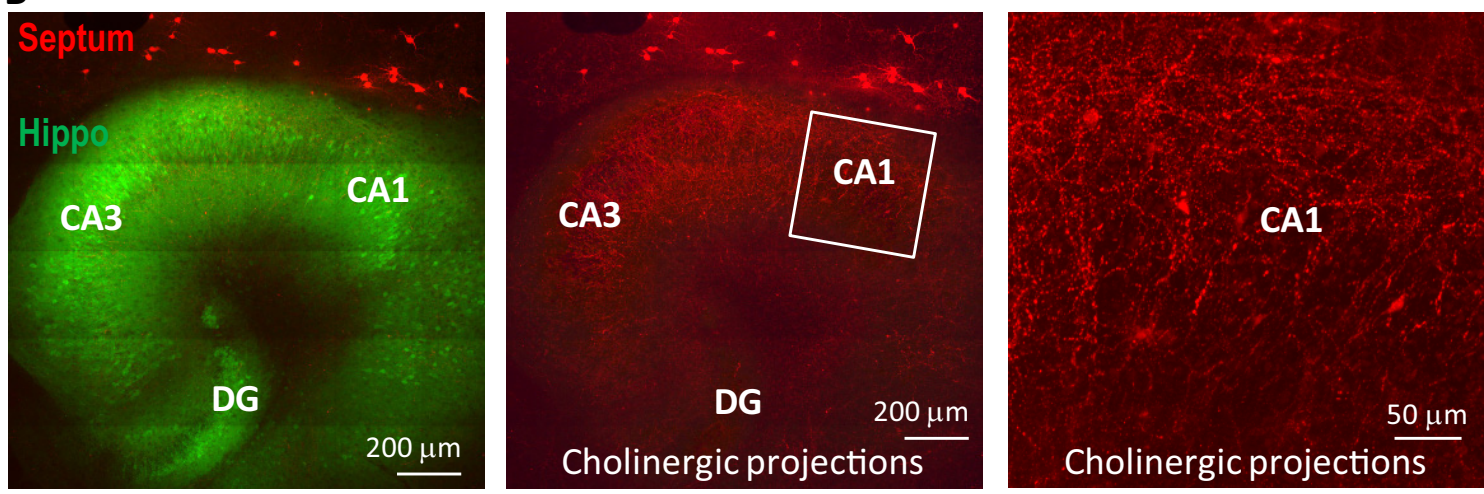

C
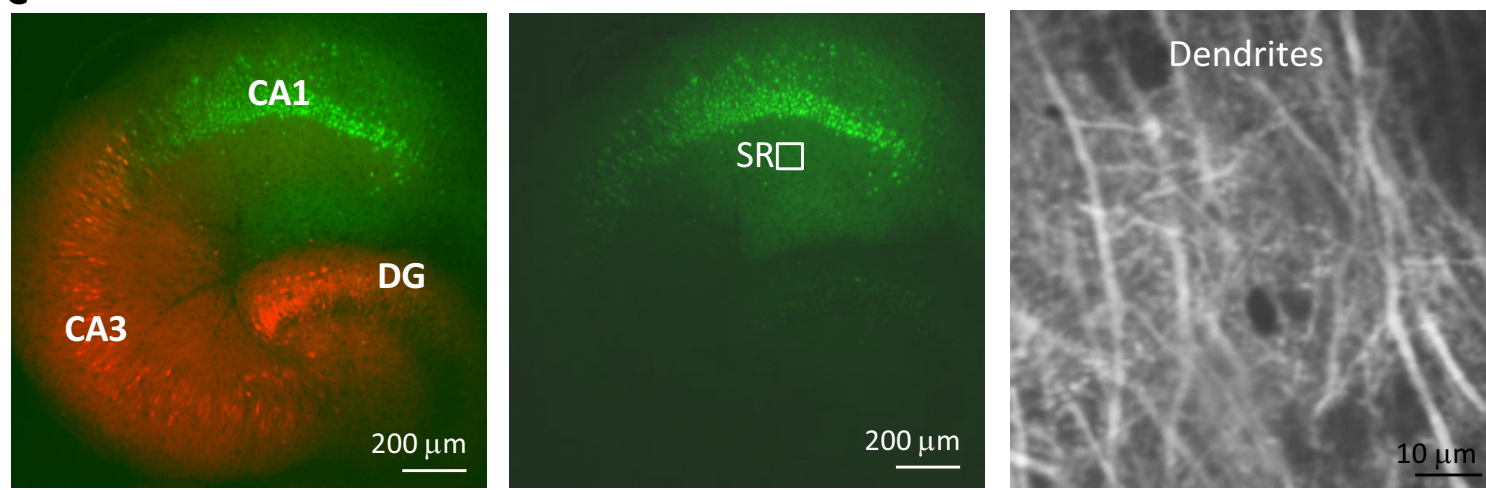

D
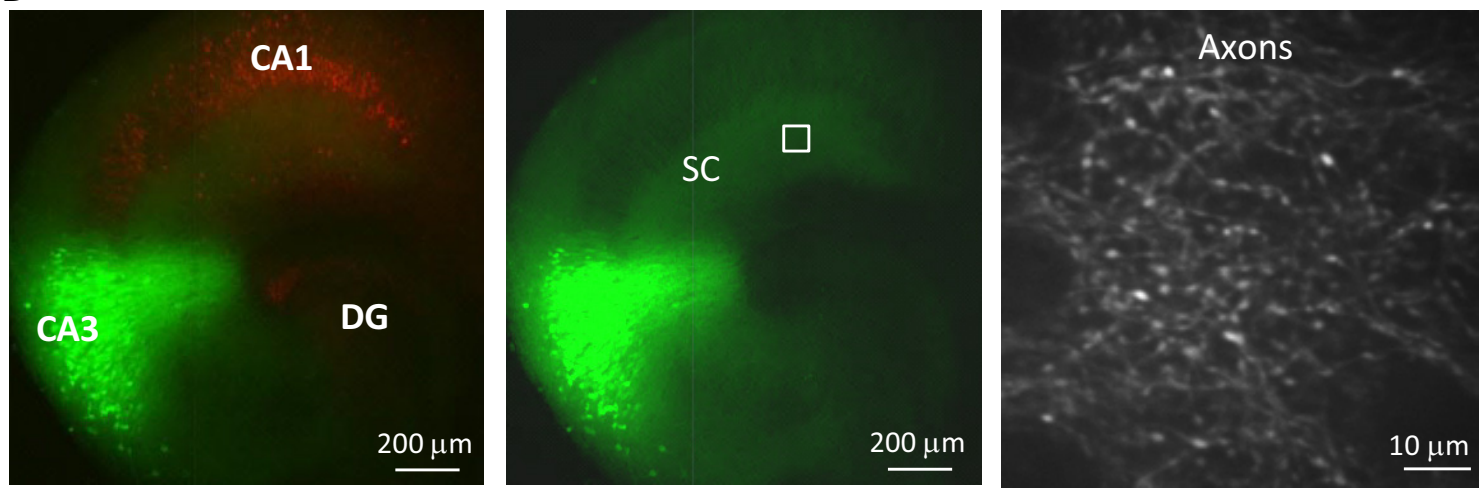

Figure 1. Monitoring neuronal synaptic activities with calcium imaging in septohippocampal slice cocultures. A, Septal tissue and hippocampus were dissected out from $300 \mu \mathrm{m}$ brain slices and placed next to each other for culture. B, Abundant cholinergic innervation (red) into the hippocampus (Hippo) (green) 7 days after coculturing. C, Genetically encoded calcium indicator GCaMP3 (green, in synapsin promoter-driven AAV) was expressed in hippocampal CA1 neurons, and the dendritic spines were monitored in CA1 SR layer (box in the middle panel) as postsynaptic activities (right panel).D, GCaMP3 was expressed in CA3 neurons, but the projecting axons were monitored in CA1 SR area as presynaptic activities (right panel). The remaining areas of the hippocampus were shown with another red fluorescent protein also driven by synapsin promoter. MS, Medial septum; DG, dentate gyrus. 
of hippocampal functions (Gahwiler and Brown, 1985; Fischer et al., 1999), even including complex network activities like theta oscillations (Fischer et al., 1999). In addition, a major advantage of using the coculture system (instead of acute hippocampal slices) is that this allows for the expression of proteins (e.g., nAChR subunits or GECIs) to restricted hippocampal subregions either in wild-type or transgenic mice (Fig. 1). We have previously shown that septal cholinergic inputs, activated either by electrical stimulation or via an optogenetic approach, can induce three types of hippocampal Schaffer collateral (SC) to CA1 synaptic plasticity, depending on the timing of the cholinergic input activation relative to the SC inputs in acute hippocampal slices (Gu and Yakel, 2011). In this study, we used GECIs to measure calcium activity changes at both presynaptic and postsynaptic sites to understand their respective contributions to the expression of synaptic plasticity (Fig. $1 C, D)$. Second, we wanted to express the $\alpha 7$ nAChR subunit to either presynaptic or postsynaptic sites (or both) in $\alpha 7 \mathrm{nAChR}$ knock-out slices to dissect out the roles of presynaptic and postsynaptic $\alpha 7 \mathrm{nAChRs} \mathrm{in}$ inducing the $\alpha 7 \mathrm{nAChR}$-dependent LTP and STD. To do this, medial septum tissues and hippocampi were dissected out from brain slices and placed next to each other on Transwell membranes and cultured (Fig. $1 A$ ). The following day, viruses encoding either the GECIs or the $\alpha 7 \mathrm{nAChR}$ subunit were microinjected into the hippocampal $\mathrm{CA} 1$ or $\mathrm{CA} 3$ regions, and experiments were done 7 days after viral infection to allow protein expression and cholinergic innervation into the hippocampus (Fig. $1 B-D$ ).

First we verified that the three different forms of ACh release-induced synaptic plasticity that we had previously observed in acute hippocampal slices using an optogenetic approach was well preserved with the same timing-sensitive modulation in the septohippocampal coculture system. We selectively expressed the light-activated cation channel channelrhodopsin-2 (ChR2) in cholinergic neurons by microinjecting a Cre-inducible AAV containing a double-floxed inverted ChR2 (fused with mCherry for visualization) (Tsai et al., 2009; Witten et al., 2010) to the septal tissue from ChAT-Cre transgenic mice. Extensive cholinergic innervations to the hippocampus can be observed 7 days later (Fig. 1B). Cholinergic inputs were then activated with $488 \mathrm{~nm}$ light exposure $(20 \mathrm{~ms})$ in the stratum oriens layer above the CA1 pyramidal neuron under patch-clamp recording. The SC pathway was activated via an electrode (Fig. 2A). Pairing of cholinergic fiber activation (via light exposure) with SC pathway (electrical) stimulation at three time intervals was selected to mimic the corresponding pairings of SO and SC electrical stimulation that produced the three types of synaptic plasticity previously observed in acute hippocampal slices (Gu and Yakel, 2011) (Fig. 2B). dentate gyrus.

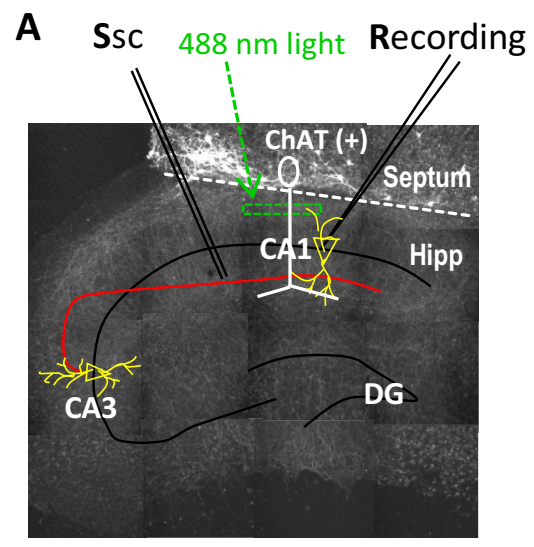

B
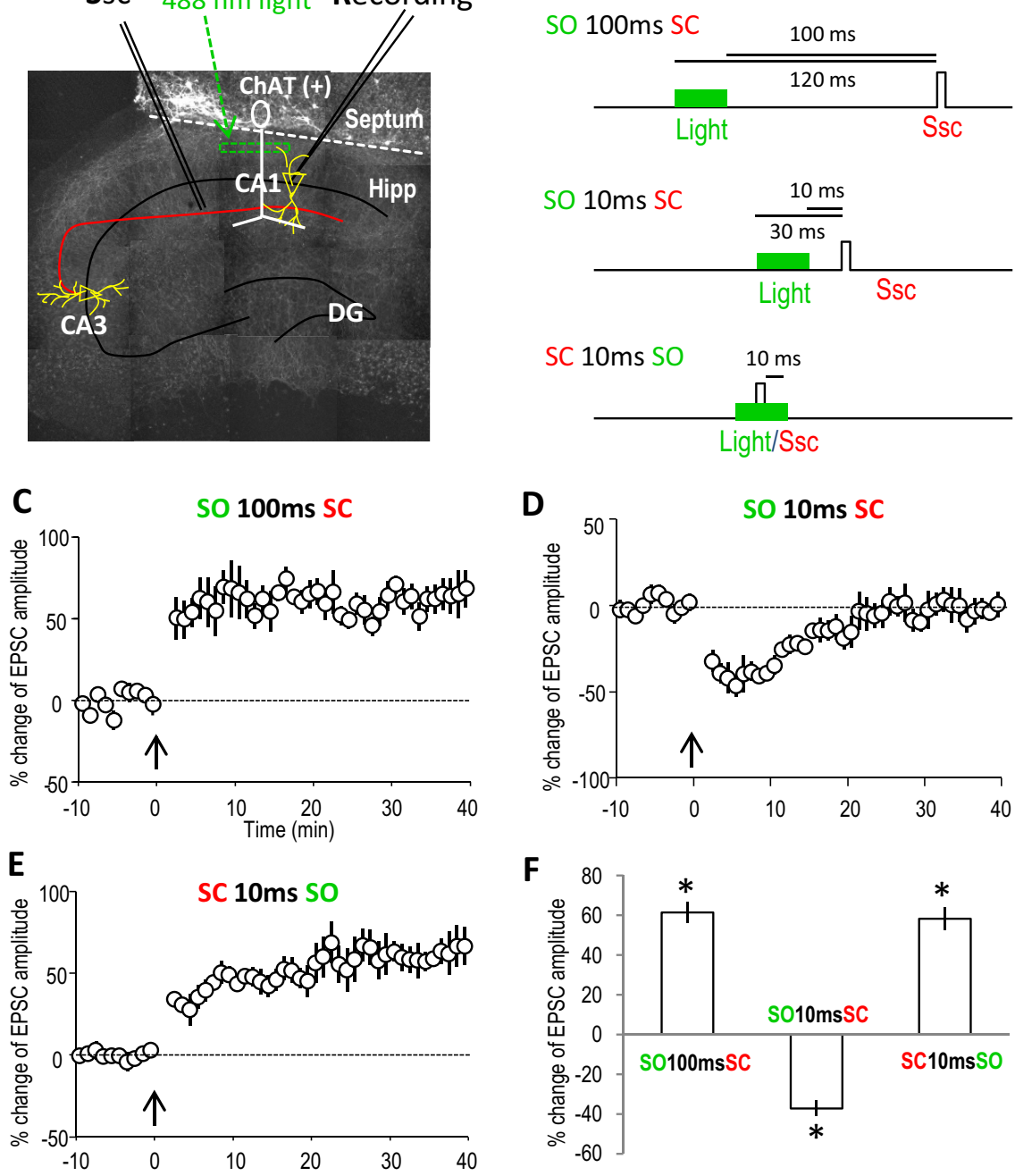

Figure 2. Optogenetically activated cholinergic inputs induce similar timing-dependent hippocampal plasticity in septohippocampal cocultures as in acute hippocampal slices. $\boldsymbol{A}$, Cholinergic projections (infected with (hR2) in a small region cholinergic (SO) and SC pathways were used to induce plasticity, as used in acute hippocampal slices previously. C, LTP was induced by optically activating the cholinergic input $100 \mathrm{~ms}$ before stimulating the SC. The pairing protocol was introduced the SC. $\boldsymbol{E}$, LTP was induced by pairing the SC 10 ms before optically activating the cholinergic input. $\boldsymbol{F}$, Bar graph showing amplitude changes of the three types of synaptic plasticity, analyzed at 30 min for LTP and 10 min for STD. ${ }^{*} p<0.001$ as compared with before pairing, Student's $t$ test, $n=5$ in each group. Ssc, Stimulation of the SC; Hipp, hippocampus; DG,

Consistent with the results from acute hippocampal slices, when the cholinergic input was activated $100 \mathrm{~ms}$ before SC stimulation, LTP was induced (Fig. 2C). When cholinergic inputs were activated $10 \mathrm{~ms}$ before SC stimulation, STD was induced (Fig. 2D). Finally, when cholinergic input was activated $10 \mathrm{~ms}$ after SC stimulation, LTP was induced (Fig. 2E). These results demonstrated that the complex cholinergic modulation of hippocampal plasticity was well preserved in the septohippocampal coculture system as in the acute hippocampal slices (Fig. 2F). Furthermore, these results reinforced our previous observation of the robust timing-dependent cholinergic control of hippocampal function by successfully duplicating it in another system. Here, we focus on these first two forms of plasticity (i.e., the LTP and STD), both of which were previously shown to be dependent on activation of the $\alpha 7 \mathrm{nAChR}$, and attempt to dissect out the individual contribution of presynaptic and postsynaptic $\alpha 7$ $\mathrm{nAChRs}$ in inducing this plasticity. 
The $\alpha 7$ nAChR-dependent LTP involves prolonged calcium activity enhancement at both presynaptic and postsynaptic sites

Initially we examined whether the $\alpha 7 \mathrm{nAChR}$-dependent LTP was due to enhanced presynaptic or postsynaptic activity. By expressing the genetically encoded calcium indicator GCaMP3 (Tian et al., 2009) in restricted hippocampal subregions, we have been able to monitor the SC stimulation-induced intracellular calcium increases in either presynaptic or postsynaptic sites during and after the induction of LTP. To measure postsynaptic activity in the CA1 region, GCaMP3 was selectively expressed in hippocampal CA1 neurons and their dendrites, and the fluorescent signal was monitored in the CA1 stratum radiatum (SR) layer (Fig. 1C). To measure presynaptic activity in the CA1 region instead, GCaMP3 was selectively expressed in CA3 neurons by viral microinjection in the CA3 region, and the fluorescent signal from the projecting axons was monitored in the CA1 SR layer (Fig. 1D). To avoid activating ChR2 while imaging GCaMP3 with $488 \mathrm{~nm}$ light, in these cases the cholinergic neurons were electrically stimulated with an electrode placed in the septal tissue (rather than using light to activate ChR2); this induced the same timing-dependent plasticity when paired with SC stimulation (data not shown).

Calcium imaging was carried out in the CA1 SR region $5 \mathrm{~s}$ before and after SC stimulation to measure the basal transient increase in intracellular calcium levels (i.e., GCaMP3 intensity increase) due to SC stimulation. When measuring the averaged fluorescence from the whole image, stimulation of the SC pathway usually induced a $5 \%$ increase over the background fluorescence, with the peak reached within $0.5 \mathrm{~s}$ and returning to basal levels within $2 \mathrm{~s}$ (Fig. $3 A$ ). This sampling was repeated every 1-2 min until stable responses were achieved (usually after about $10 \mathrm{~min}$ ), after which the pairing protocol was introduced to induce synaptic plasticity. The sampling was then carried out for another $30 \mathrm{~min}$ to monitor any changes in the SC stimulation-induced calcium responses, and then the peak amplitude of the responses at 10 and $30 \mathrm{~min}$ after the pairing protocol were compared with the basal SC responses.

When monitoring the postsynaptic calcium activity in the CA1 SR region (i.e., when GCaMP3 was expressed in CA1 neurons and the dendrites were monitored), the SC stimulation-induced response amplitudes were significantly increased after pairing the cholinergic input $100 \mathrm{~ms}$ before SC pathway stimulation ( $43 \pm 8$ and $58 \pm 6 \%$ increase at 10 and $30 \mathrm{~min}$, respectively, $n=6$; Fig. $3 A, B, E$ ). This increase lasted for at least $30 \mathrm{~min}$, demonstrating that a protocol that induces LTP at CA1 synapses induces prolonged increases in postsynaptic calcium activity. When monitoring presynaptic calcium activity in the same region (i.e., when the GCaMP3 was expressed in the CA3 neurons and the projecting SC axons were monitored in the CA1 SR region), SC stimulation-induced response amplitudes were also increased after pairing cholinergic input 100 ms before SC pathway stimulation ( $54 \pm 3$ and $41 \pm 7 \%$ increase at 10 and $30 \mathrm{~min}$, respectively, $n=6$; Fig. $3 C-E$ ), also demonstrating that the LTP-inducing protocol significantly increases presynaptic calcium activity for a prolonged period of time. These presynaptic data are significant, as our previous work had suggested that there was only a transient increase in presynaptic activity when using the paired-pulse ratio (PPR) as an indicator (Gu and Yakel, 2011).

\section{Both presynaptic and postsynaptic $\alpha 7 \mathrm{nAChRs}$ are required to induce the $\alpha 7 \mathrm{nAChR}$-dependent LTP}

We previously showed that the $\alpha 7$ nAChR-dependent LTP was likely due to a postsynaptic effect, since it appeared to require the activation of the NMDAR and prolongation of the NMDAR- mediated calcium transients in the spines and GluR2-containing AMPAR synaptic insertion. To examine the contributions of presynaptic and/or postsynaptic $\alpha 7$ receptors to plasticity in more detail, we used a strategy of restoring the expression of the $\alpha 7$ nAChR subunit to slices obtained from $\alpha 7$ nAChR knock-out mice; in these mice, this form of LTP was absent. Initially, we verified that in septohippocampal cocultures from $\alpha 7 \mathrm{nAChR}$ knock-out mice (the septal tissue providing cholinergic inputs was from wild-type mice), the pairing protocol that normally would induce the $\alpha 7 \mathrm{nAChR}$-dependent LTP, failed to induce any significant prolonged increase in calcium signals at either postsynaptic or presynaptic sites $(-6 \pm 4$ and $-3 \pm 5 \%$ change at 30 min, respectively, $n=5$; Fig. $3 E$ ).

Next, we then restored the $\alpha 7$ nAChR (Séguéla et al., 1993) to either postsynaptic (by expressing the $\alpha 7 \mathrm{nAChR}$ along with GCaMP3 into CA1 neurons) or presynaptic sites (by expressing the $\alpha 7$ receptor into CA3 neurons in $\alpha 7 \mathrm{nAChR}$ knock-out slices). The $\alpha 7 \mathrm{nAChR}$ was also subcloned to the AAV vector under the synapsin promoter. The restricted localization of $\alpha 7$ nAChRs and GCaMP3 to either CA1 or CA3 was verified with the GCaMP3 expression pattern before imaging experiments. When the $\alpha 7$ receptor was restored in the postsynaptic CA1 neurons only, the SC stimulation-induced GCaMP3 responses were transiently increased in the postsynaptic sites after the pairing $(44 \pm$ $5 \%$ and $-1 \pm 2 \%$ change at 10 and $30 \mathrm{~min}$, respectively, $n=5$; Fig. $4 A, B, E)$. Similarly, when the $\alpha 7 \mathrm{nAChR}$ was restored in the presynaptic CA3 neurons only, the SC stimulation-induced GCaMP3 responses were also transiently increased in the presynaptic sites after the pairing $(50 \pm 7 \%$ and $4 \pm 4 \%$ increase at 10 and $30 \mathrm{~min}$, respectively, $n=5$; Fig. $4 C-E$ ). When we expressed the $\alpha 7 \mathrm{nAChR}$ in both CA1 and CA3 neurons in the same slice (i.e., both postsynaptically and presynaptically), this restored the LTP that was seen in wild-type slices (i.e., a prolonged enhancement in the SC stimulation-induced GCaMP3 responses both presynaptically and postsynaptically; $31 \pm 6$ and $70 \pm 7 \%$ increase at $30 \mathrm{~min}$, respectively, $n=5$; Fig. $4 E$ ) These data demonstrate that both presynaptic and postsynaptic $\alpha 7 \mathrm{nAChRs}$ play a role in regulating synaptic activities and that both presynaptic and postsynaptic $\alpha 7$ receptors must be present to induce the $\alpha 7$ nAChR-dependent LTP. Furthermore these findings suggest that coordination between the presynaptic and postsynaptic modulation is required to induce this form of LTP.

\section{Both presynaptic and postsynaptic $\alpha 7 \mathrm{nAChRs}$ are required to induce the $\alpha 7 \mathrm{nAChR}$-dependent STD}

We have previously shown that the STD induced by pairing cholinergic input stimulation $10 \mathrm{~ms}$ before SC pathway stimulation was also mediated by the $\alpha 7 \mathrm{nAChR}$, although a presynaptic mechanism was previously suggested from the change in the paired-pulse ratio. Therefore, we tested whether a presynaptic mechanism alone is sufficient to explain this STD. In wild-type cocultured slices, we observed a transient decrease in the SC stimulation-induced GCaMP3 responses at both postsynaptic $(-39 \pm 5$ and $8 \pm 5 \%$ change at 10 and $30 \mathrm{~min}$, respectively, $n=5$; Fig. $5 A, B, E)$ and presynaptic sites $(-37 \pm 6$ and $-3 \pm 3 \%$ change at 10 and $30 \mathrm{~min}$, respectively, $n=$ 5; Fig. 5C-E). Furthermore, both responses were abolished in $\alpha 7$ nAChR knock-out slices (Fig. 5E). When we restored the $\alpha 7 \mathrm{nAChR}$ to either the presynaptic ( $5 \pm 7 \%$ change at $10 \mathrm{~min}, n=5$; Fig. $5 F$ ) or the postsynaptic ( $6 \pm 7 \%$ change at $10 \mathrm{~min}, n=5$; Fig. $5 F)$ sites alone (in $\alpha 7 \mathrm{nAChR}$ knock-out slices), we could not restore the STD (Fig. $5 F$ ); restoring the $\alpha 7$ receptor to both presynaptic and postsynaptic sites in the same slice was required to restore the STD in the $\alpha 7$ knock-out slices (Fig. 5F). This suggests that a presynaptic mecha- 

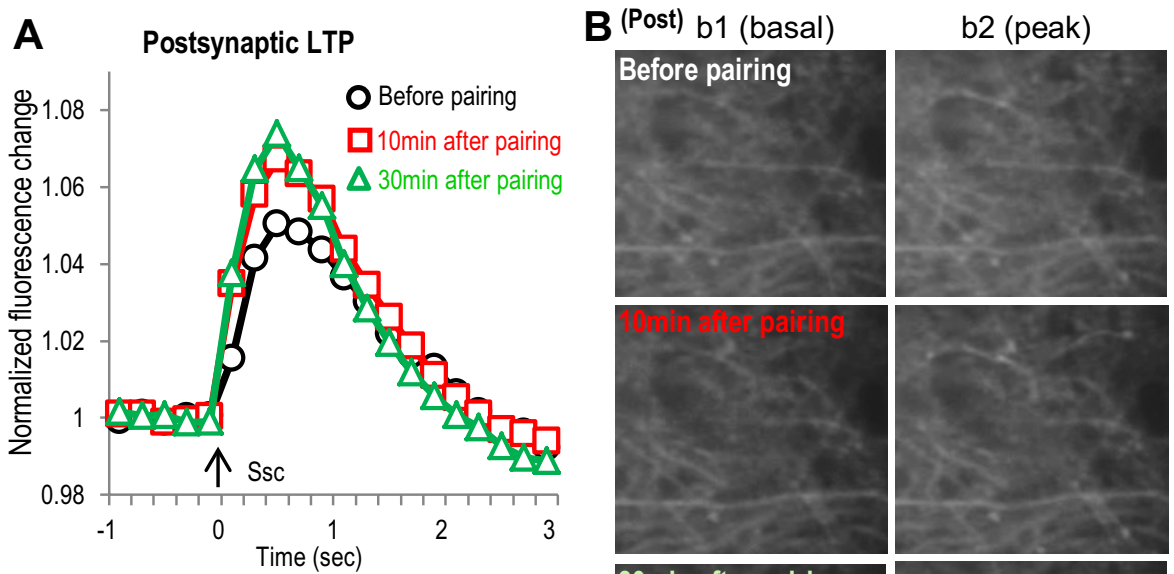

b3 (b2-b1)
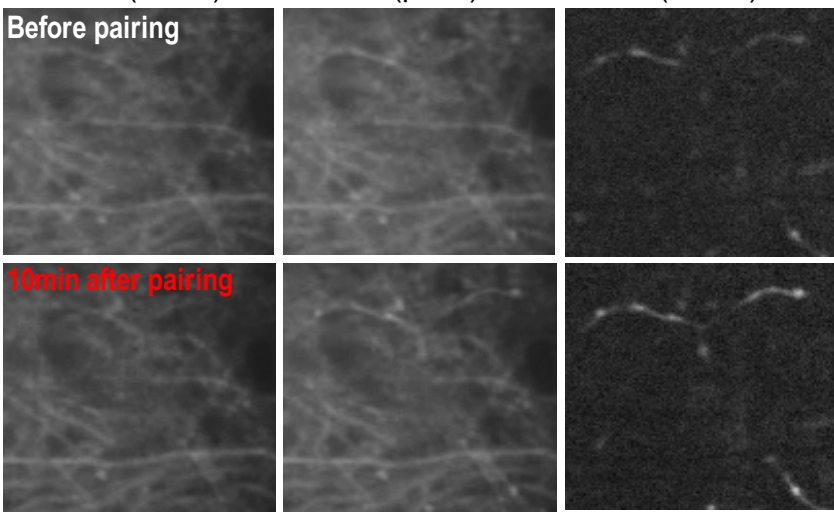

\section{C}
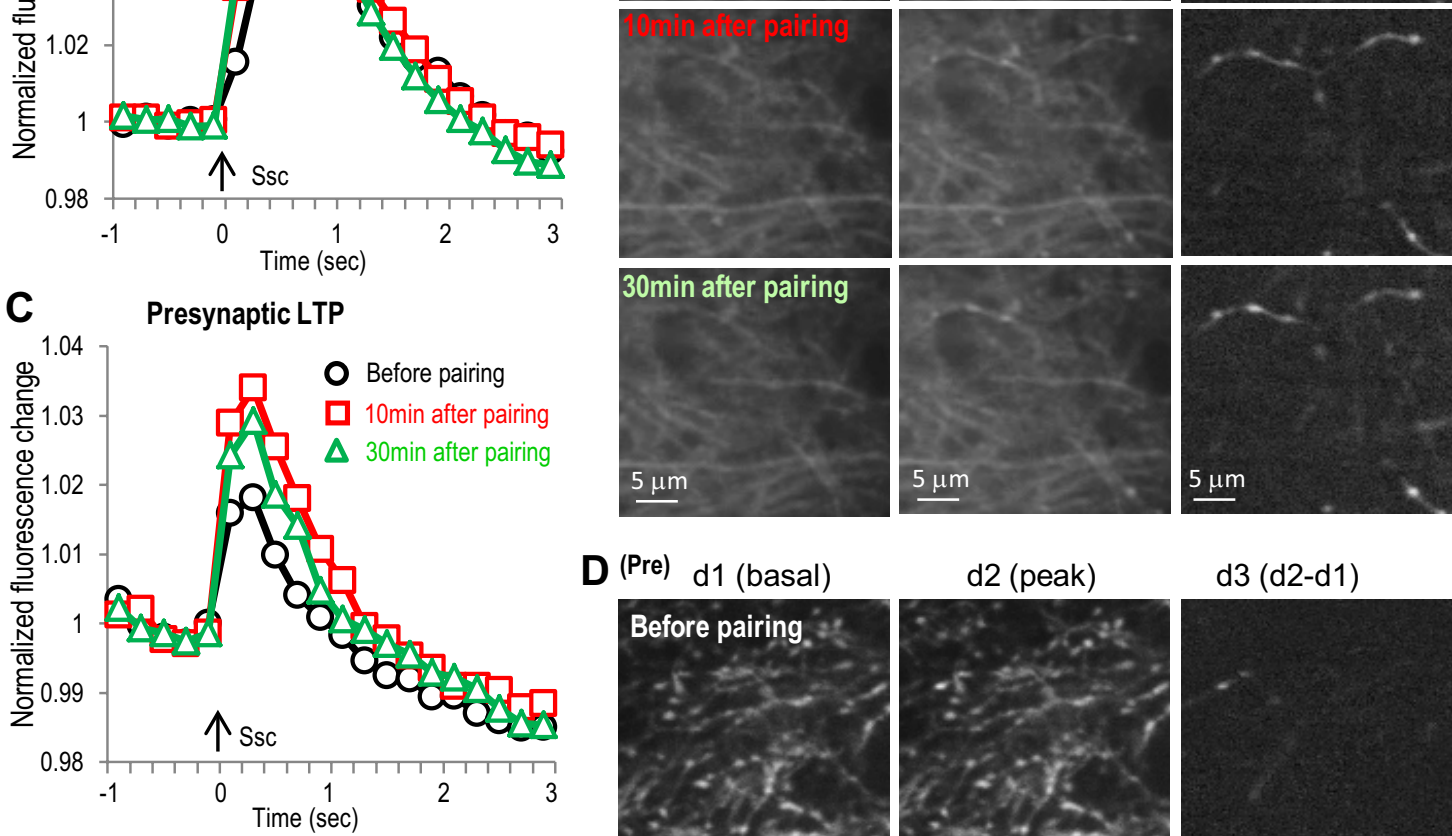

D (Pre) d1 (basal)
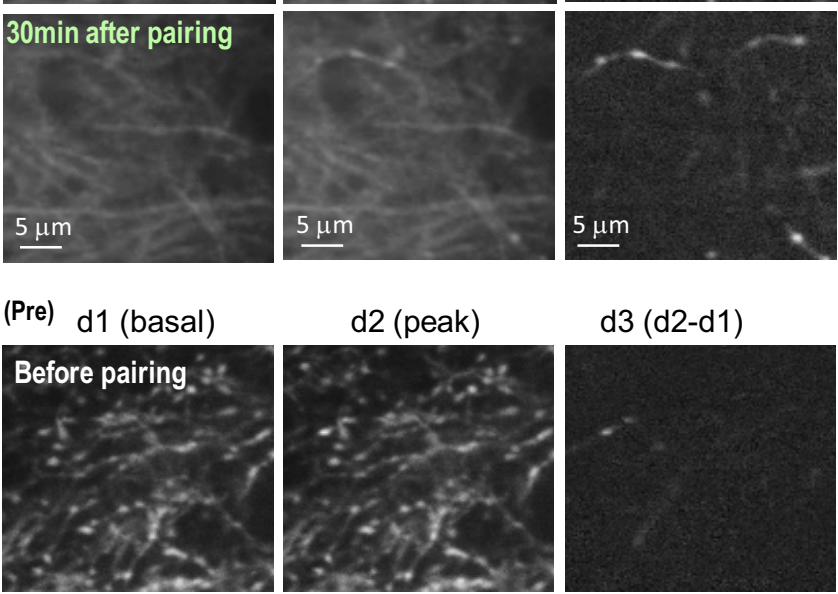

\section{E}
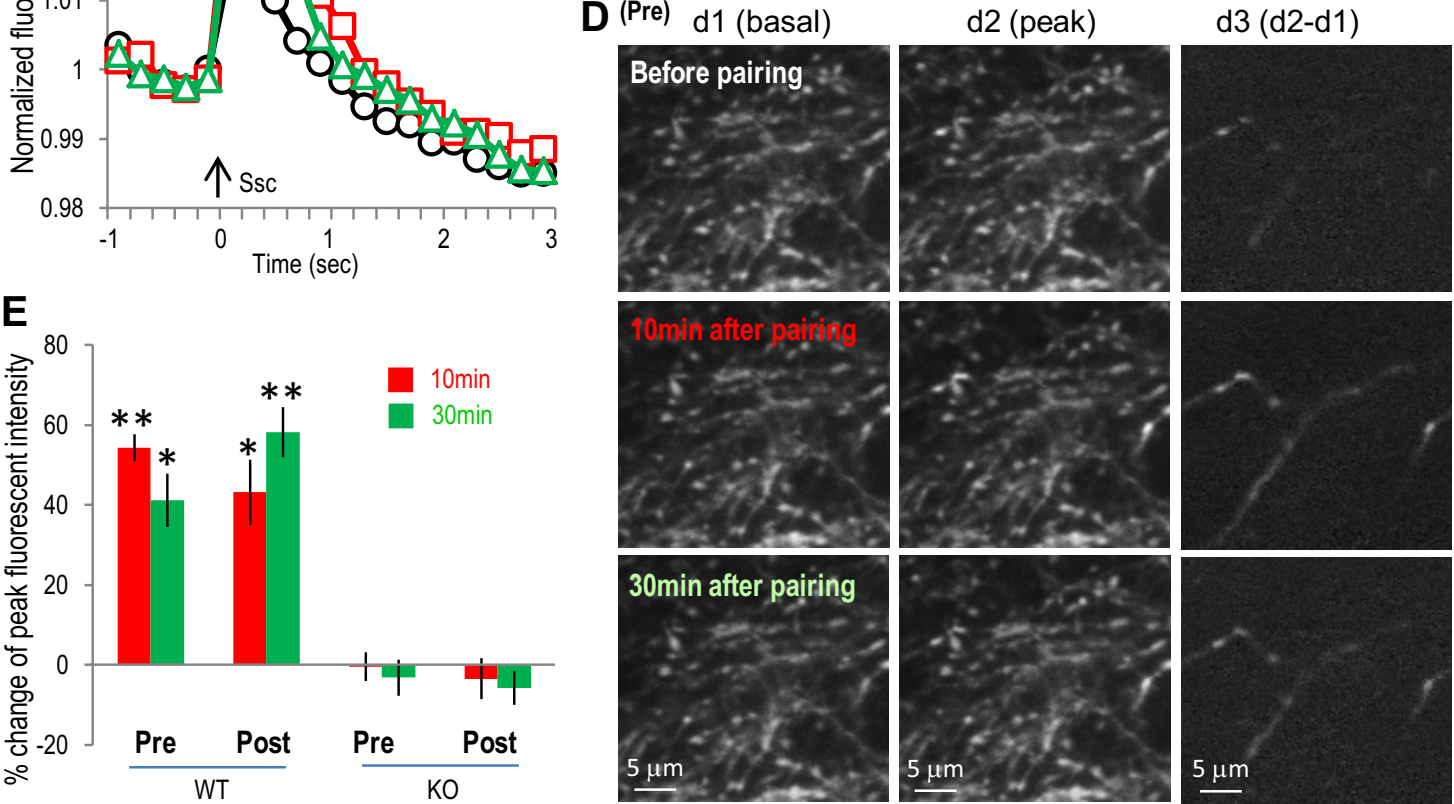

Figure 3. The $\alpha 7$ nAChR-mediated LTP involves prolonged calcium activity enhancement in both presynaptic and postsynaptic sites in septohippocampal slice cocultures. LTP was induced by pairing SO pathway 100 ms before SC pathway in septohippocampal slice cocultures. A, Normalized SC pathway stimulation-induced postsynaptic GCaMP3 responses showing the prolonged enhancement of postsynaptic calcium activities after the SO pathway pairing protocol. SC pathway was stimulated at the time point of $0 \mathrm{~s}$ as indicated by the arrow. $\boldsymbol{B}$, Images of SC pathway-induced postsynaptic calcium imaging from different time points before or after pairing protocol. C, Normalized presynaptic GCaMP3 responses showing the prolonged enhancement after the $S 0$ pathway pairing protocol. D, Images of presynaptic calcium imaging from different time points before or after pairing protocol. $\boldsymbol{E}$, Bar graph showing the prolonged postsynaptic (Post) and presynaptic (Pre) responses during LTP; both were abolished in $\alpha 7$ nAChR knock-out slices. ${ }^{*} p<0.01,{ }^{* *} p<0.001$, as compared with before pairing, Student's $t$ test, $n=6$ in each group. Ssc, Stimulation of the SC; WT, wild type.

nism alone is not sufficient to induce this form of STD, again further implicating some type of coordination between presynaptic and postsynaptic sites.

The expression of functional $\alpha 7 \mathrm{nAChRs}$ in pyramidal neurons was verified by directly measuring choline (10 $\mathrm{mM})$-induced $\alpha 7 \mathrm{nAChR}$ currents (Fayuk and Yakel, 2004). The $\alpha 7 \mathrm{nAChR}$ currents were induced in all of the CA1 and CA3 pyramidal neurons we tested (8-12 neurons for either group), with peak amplitudes of $\sim 50-100 \mathrm{pA}$ that were completely blocked by the $\alpha 7$-selective antagonist methyllycaconitine (MLA) (10 nM); choline-induced responses were absent in $\alpha 7 \mathrm{nAChR}$ knock-out slices (Fig. 6A,B). Virus-introduced $\alpha 7 \mathrm{nAChRs}$ (fused and visualized with yellow fluorescent protein, YFP, in AAV virus under the synapsin promoter) restored $\alpha 7 \mathrm{nAChR}$ currents in both CA1 and CA3 neurons from $\alpha 7 \mathrm{nAChR}$ knock-out slices, with peak amplitudes not significantly different than those from wild-type slices (Fig. 6A,B) in both CA1 and CA3 neurons. Expression of the reporter YFP alone (in AAV under the synapsin promoter) did not result in any $\alpha 7 \mathrm{nAChR}$ currents in knock-out slices and had no obvious effects in wild-type slices (data were combined with the respective knock-out and wild-type groups as shown in Fig. 6B). 
A

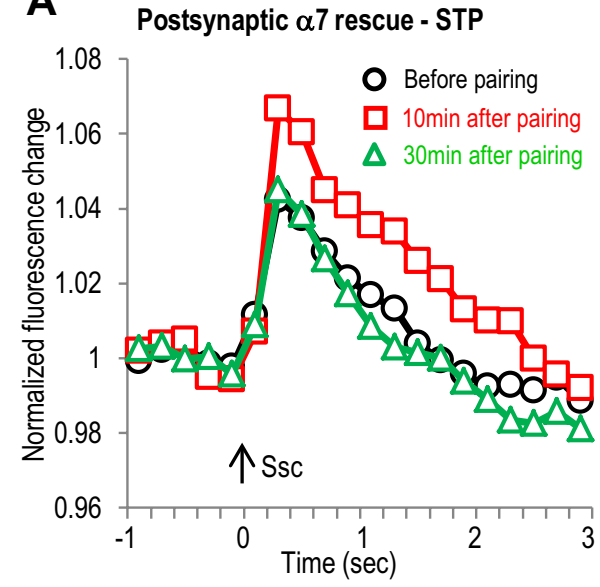

C

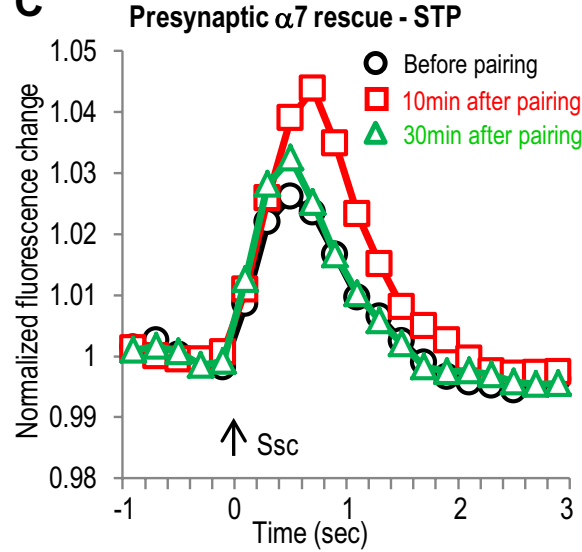

E

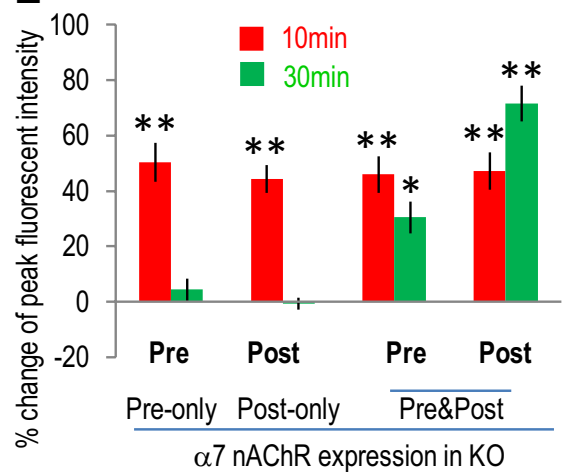

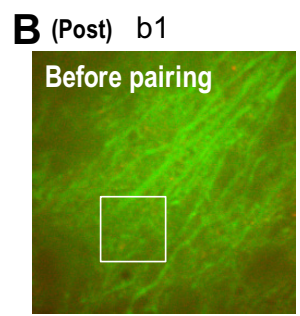
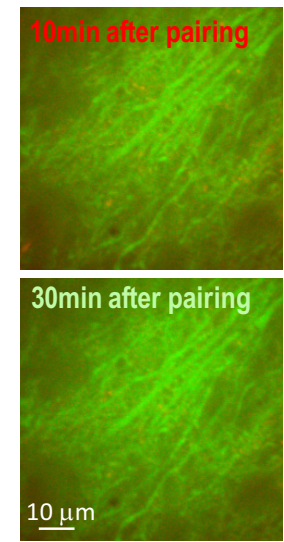

D (Pre) d1
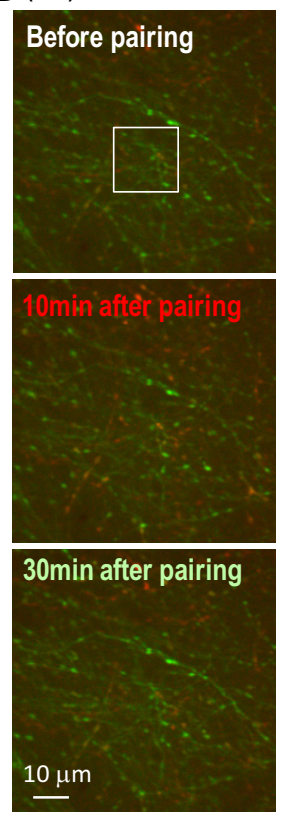

b2 (the Ssc response)
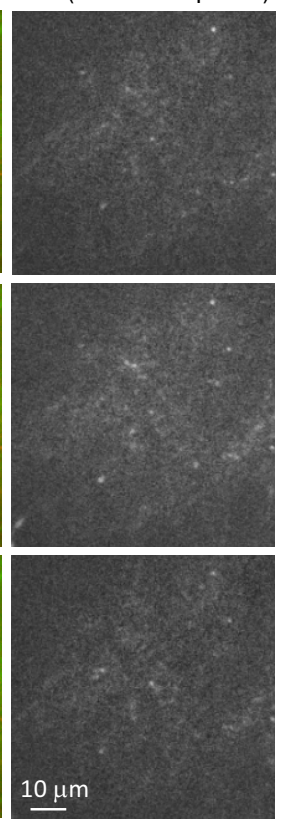

d2 (the Ssc response)
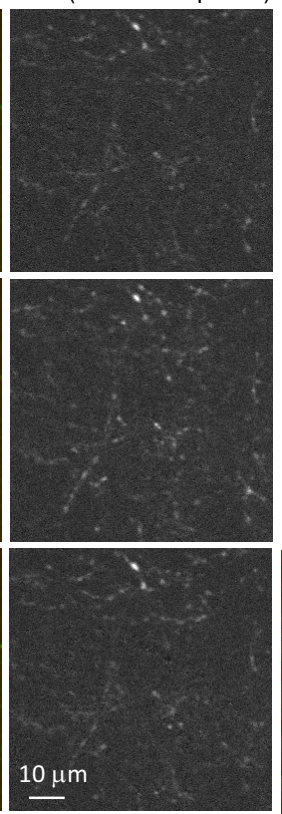

b3 (inset in b1)

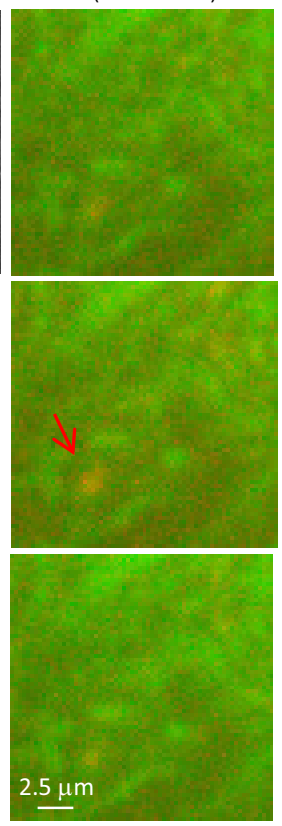

d3 (inset in d1)
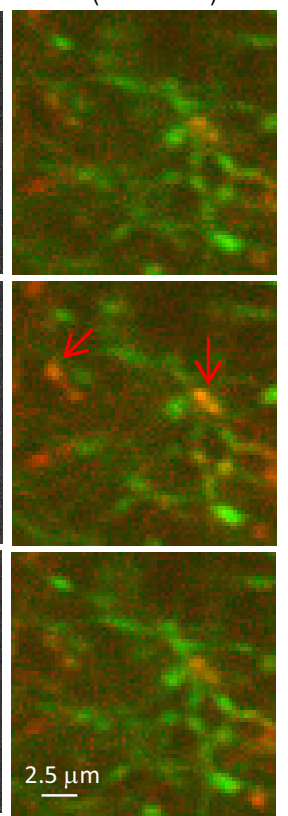

Figure 4. Expression of $\alpha 7 \mathrm{nAChR}$ in postsynaptic or presynaptic sites in $\alpha 7 \mathrm{nAChR} \mathrm{KO}$ slice only induced transient activity enhancement in postsynaptic or presynaptic sites, respectively. $\mathrm{A}$, $\alpha 7$ nAChR was coexpressed with GCaMP3 in hippocampal CA1 area of $\alpha 7 \mathrm{nAChR} \mathrm{knock-out} \mathrm{mice} \mathrm{(where} \mathrm{the} \alpha 7 \mathrm{nAChR-dependent} \mathrm{LTP} \mathrm{was} \mathrm{abolished)} \mathrm{as} \mathrm{postsynaptic} \mathrm{rescue} \mathrm{of} \alpha 7 \mathrm{nAChR}$. Normalized postsynaptic GCaMP3 responses show transient enhancement after the $\mathrm{SO}$ pathway pairing protocol. $\boldsymbol{B}$, Images of postsynaptic calcium imaging from different time points before or after pairing protocol, with left panels showing the original images, middle panel showing the active calcium responses to SC stimulation, and right panels showing some potential dendritic spines. The potentiation was present in most of the dendrites responsive to SC stimulation (middle panels). $C, \alpha 7 \mathrm{nAChR}$ was coexpressed with GCaMP3 in hippocampal CA3 area as presynaptic rescue. Normalized presynaptic $G C a M P 3$ responses show transient enhancement of presynaptic calcium activities after the $S 0$ pathway pairing protocol. $\boldsymbol{D}$, Images of presynaptic calcium imaging from different time points before or after pairing protocol, with individual panels similar as described in B.E, Bar graph showing the requirement of both presynaptic and postsynaptic presence of $\alpha 7 \mathrm{nAChRs}$ to induce LTP. Red signals (in $\boldsymbol{B}$ and D) show theSCstimulation-induced calcium increases (also shown separately in the middle panels) over the basal signals before stimulation (green). Arrows indicate some synaptic sites with significant transient calcium increase. ${ }^{*} p<0.01,{ }^{* *} p<0.001$, as compared with before pairing, Student's $t$ test, $n=5$ in each group. Ssc, Stimulation of the SC.

To further verify the direct involvement of postsynaptic $\alpha 7$ nAChRs in CA1 pyramidal neurons in this plasticity, we compared the plasticity in individual $\alpha 7 \mathrm{nAChR}$-positive (fused with YFP) and neighboring $\alpha 7$ nAChR-negative CA1 pyramidal neurons from the knock-out rescue (both CA1 and CA3 rescue) slices with whole-cell patch clamp. Consistent with the GCaMP3 imaging data, both LTP and STD were induced in $\alpha 7 \mathrm{nAChR}-$ positive CA1 pyramidal neurons (Fig. $6 C-F$ ). However, only STP was induced in $\alpha 7 \mathrm{nAChR}$-negative CA1 pyramidal neurons with the LTP pairing protocol, and no plasticity was induced with the STD protocol (Fig. 6C-F), which is consistent with the presynaptic-only rescue imaging data. These results strongly suggest the direct involvement of $\alpha 7 \mathrm{nAChRs}$ in postsynaptic CA1 pyramidal neurons in inducing either LTP or STD. 

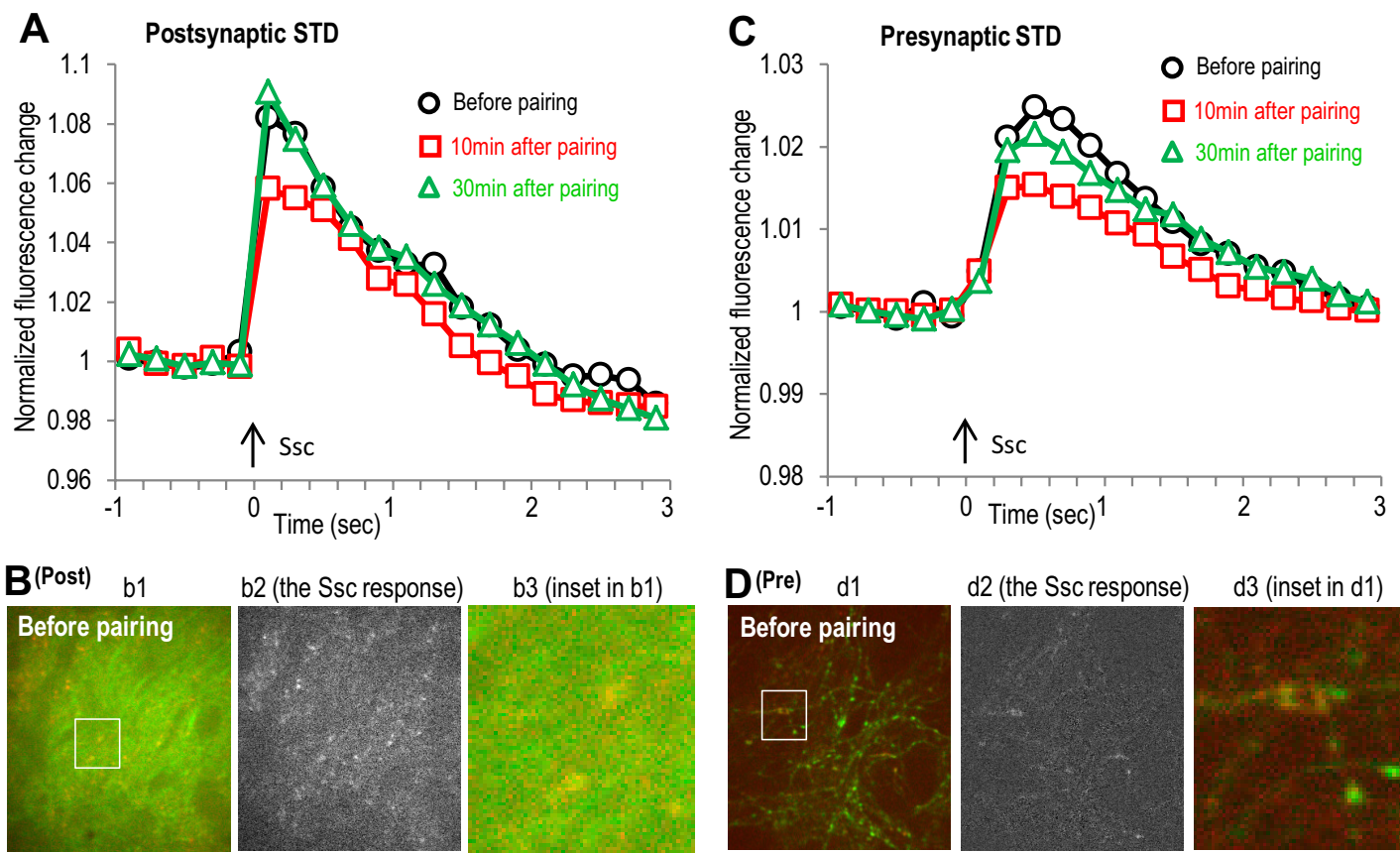

d3 (inset in d1)
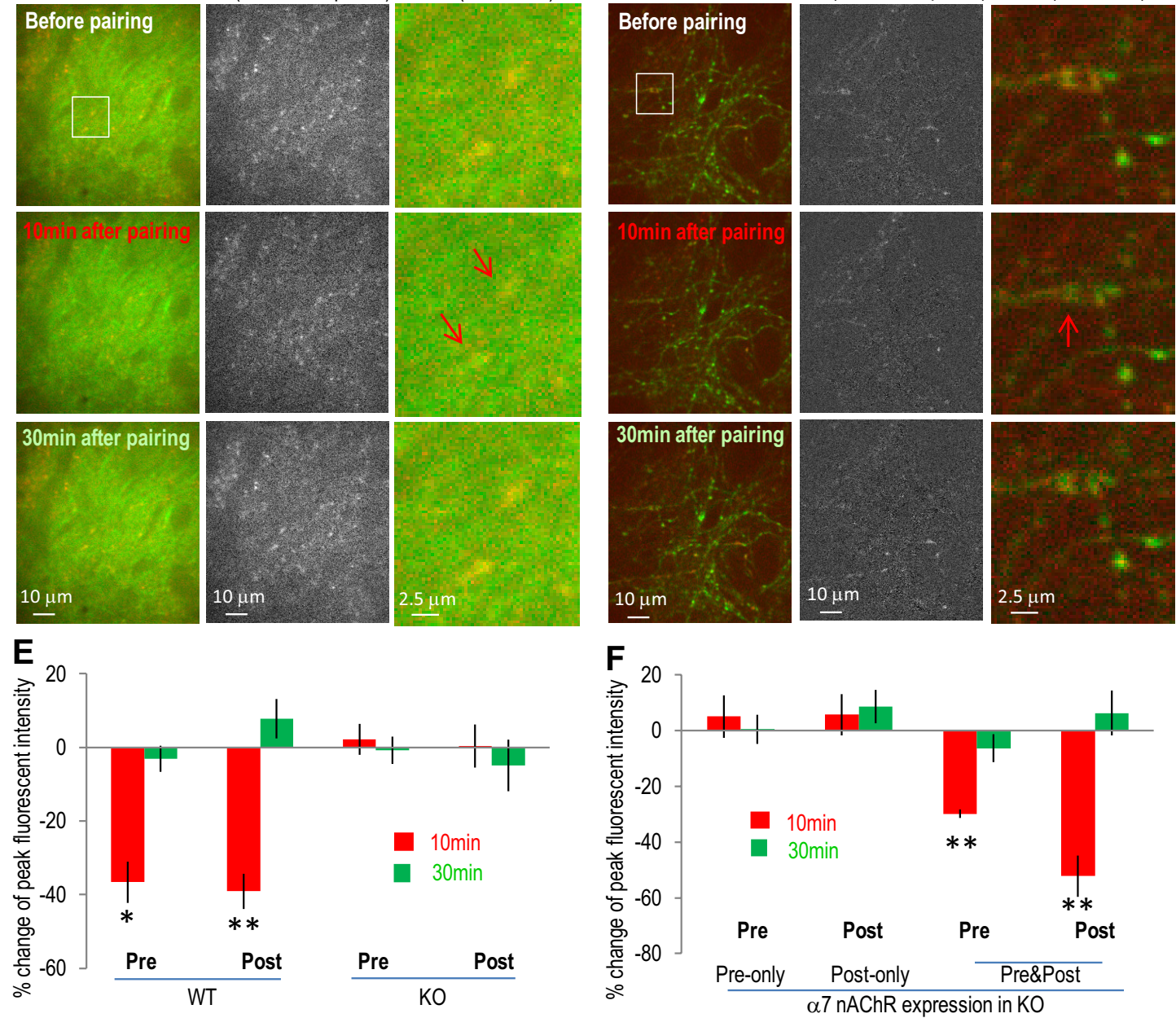

Figure 5. The $\alpha 7 \mathrm{nAChR}$-mediated STD involves transient calcium activity depression in both postsynaptic and presynaptic sites. The $\alpha 7 \mathrm{nAChR}$-dependent STD was induced by pairing SO $10 \mathrm{~ms}$ before SC. A, Normalized postsynaptic GCaMP3 responses showing the transient depression of postsynaptic calcium activities after the $S 0$ pathway pairing protocol. $\boldsymbol{B}$, Images of postsynaptic (Post) calcium imaging from different time points before or after pairing protocol, with individual panels similar as described in Figure $4 B$. C, Normalized presynaptic GCaMP3 responses showing the transient depression after the $S 0$ pathway pairing protocol. $\boldsymbol{D}$, Images of presynaptic (Pre) calcium imaging from different time points before or after pairing protocol. $\boldsymbol{E}, \boldsymbol{F}$, Bar graphs showing that the presence of $\alpha 7 \mathrm{nAChRs}$ at both presynaptic and postsynaptic sites was required to induce the STD. Red signals (in $\boldsymbol{B}$ and $\boldsymbol{D}$ ) show the SC stimulation-induced calcium increases (also shown separately in the middle panels) over the basal signals before stimulation (green). Arrows indicate some synaptic sites with significant transient calcium decrease. ${ }^{*} p<0.01,{ }^{* *} p<0.001$, as compared with before pairing, Student's $t$ test, $n=5$ in each group. Ssc, Stimulation of the SC.

Differential presynaptic and postsynaptic mechanisms in the expression of both $\alpha 7 \mathrm{nAChR}$-dependent LTP and STD revealed with dual-color GECI imaging

Although the $\alpha 7 \mathrm{nAChR}$ rescue experiments suggest that the induction of both the $\alpha 7 \mathrm{nAChR}$-dependent LTP and STD requires coordination between presynaptic and postsynaptic activities, the similar time courses between the presynaptic and postsynaptic changes could suggest that both of these two forms of plasticity were expressed primarily via presynaptic mechanisms; the observed postsynaptic change during the plasticity expression may simply be the result of presynaptic modulation. However, we did see some differences in the time course of presynaptic and post- 
A

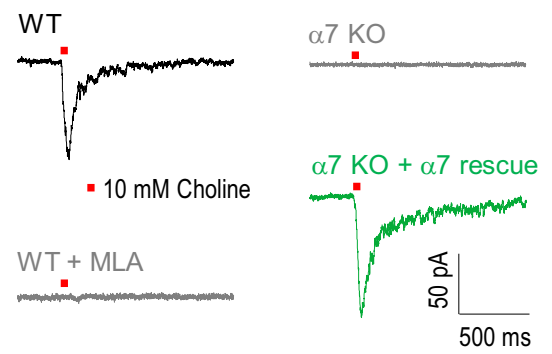

C

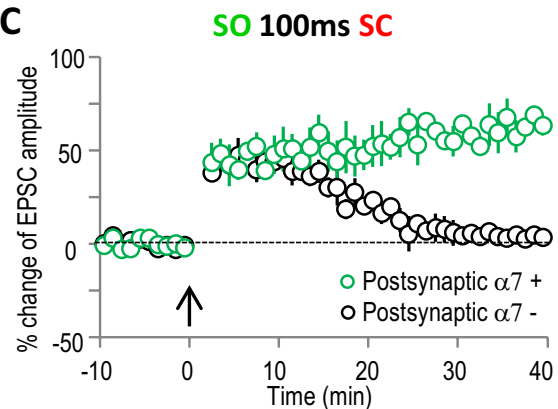

E

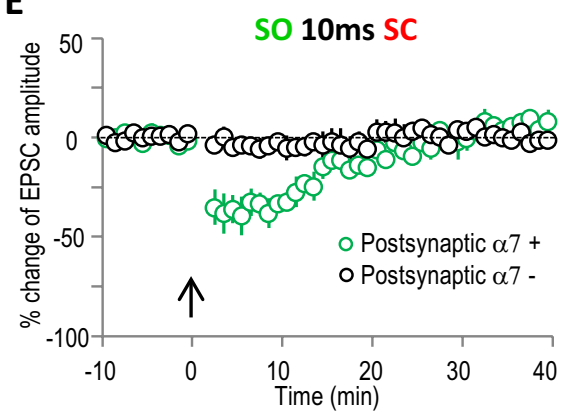

B

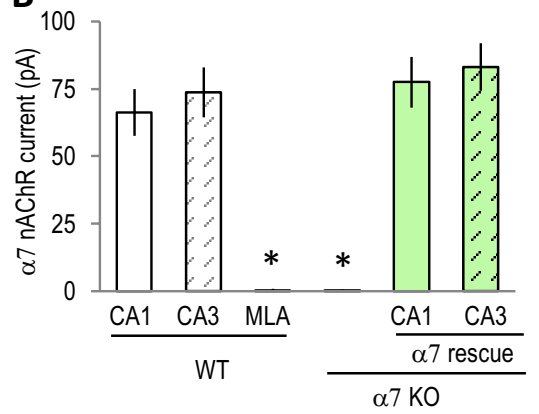

D

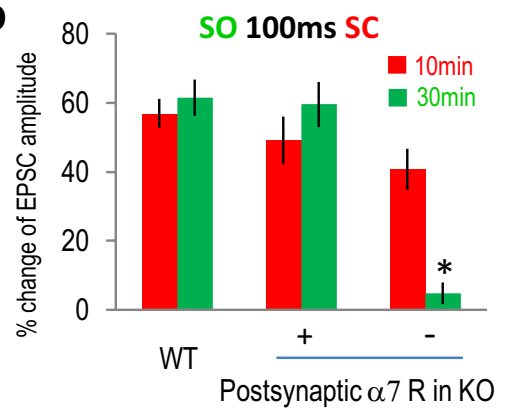

$\mathbf{F}$

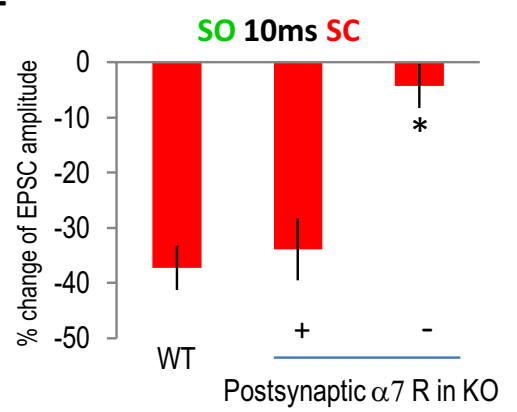

We expressed green GCaMP3 in CA3 neurons and red R-GECO1 in CA1 neurons in the same slices to observe green presynaptic and red postsynaptic activities simultaneously while monitoring in the CA1 SR region. With this dual-color imaging, we found that in many cases the activity changes in the presynaptic and postsynaptic sites were not correlated for either LTP or STD. For the example shown in Figure $7 A-D$ during the $\alpha 7$ nAChR-dependent LTP, both presynaptic and postsynaptic activities were increased at 10 and $30 \mathrm{~min}$ after the pairing protocol. However, while the postsynaptic increase was similar to presynaptic increase at $10 \mathrm{~min}$, it was significantly larger at 30 $\min$ (Fig. $7 C$ ). The ratio of postsynaptic to presynaptic response was significantly larger at $30 \mathrm{~min}$ than that at $10 \mathrm{~min}$ $(1.46 \pm 0.09$ at $30 \mathrm{~min}$ versus $0.82 \pm 0.06$ at $10 \mathrm{~min}, n=5, p<0.001, t$ test), suggesting that at least the later stage postsynaptic increase was not mainly driven by an increased presynaptic release. Instead, the data suggest that an independent postsynaptic mechanism is driving the expression of later stage LTP.

Similarly, differential modulation of presynaptic and postsynaptic activities was seen in the expression of STD. As shown in Figure 7E- $G$ during the $\alpha 7 \mathrm{nAChR}$-dependent STD, both presynaptic and postsynaptic activities were similarly decreased at 10 $\mathrm{min}$; however, they were significantly different at $30 \mathrm{~min}$. The postsynaptic to presynaptic ratio were significantly changed at 30 min as compared with that at $10 \mathrm{~min}$ $(-1.52 \pm 0.32$ at 30 min versus $1.2 \pm 0.04$ at $10 \min , n=5, p<0.001, t$ test). These results again strongly suggest the existence of an independent postsynaptic mechanism during the expression of STD.

In summary, our data show that both presynaptic and postsynaptic mechanisms contributed to the expression of the $\alpha 7$ nAChR-dependent LTP and STD.

synaptic changes during either LTP or STD (especially during LTP). For example, the increase at $30 \mathrm{~min}(58 \%)$ was larger than that at $10 \mathrm{~min}(43 \%)$ at postsynaptic sites, but smaller $(41 \%$ at 30 min versus $54 \%$ at $10 \mathrm{~min}$ ) at presynaptic sites during the LTP (Fig. 3E). Another important issue is that the presynaptic and postsynaptic activities observed above were not from the same slices, since the recordings were done separately. To clarify this question, we expressed two differently colored GECIs (green GCaMP3 and red calcium indicator R-GECO1) (Tian et al., 2009; Zhao et al., 2011) in the same slices, one at presynaptic sites and the other at postsynaptic sites. In this way we could observe the presynaptic and postsynaptic activities in the same slice simultaneously during the expression of either LTP or STD. A high degree of correlation between the changes in the presynaptic and postsynaptic activities would suggest a major role for the presynaptic sites in the expression of either form of plasticity.
More importantly, we have provided direct evidence that the $\alpha 7$ nAChR-dependent modulation of both presynaptic and postsynaptic sites is required to induce both of these forms of plasticity. Therefore, we conclude that cholinergic inputs, through activation of $\alpha 7 \mathrm{nAChRs} \mathrm{located} \mathrm{at} \mathrm{both} \mathrm{synaptic} \mathrm{sites,} \mathrm{coordinate} \mathrm{presynaptic}$ and postsynaptic activities to induce synaptic plasticity.

\section{Discussion}

Previously, we have shown that activating the septal cholinergic terminals in acute hippocampal slices induced three different types of hippocampal SC to CA1 synaptic plasticity, depending on the precise timing of cholinergic stimulation relative to the SC input (Gu and Yakel, 2011). Two of these forms of plasticity, including long-term potentiation and short-term depression, were dependent on the activation of the $\alpha 7 \mathrm{nAChR}$. Here, we have directly monitored the presynaptic and postsynaptic activity 

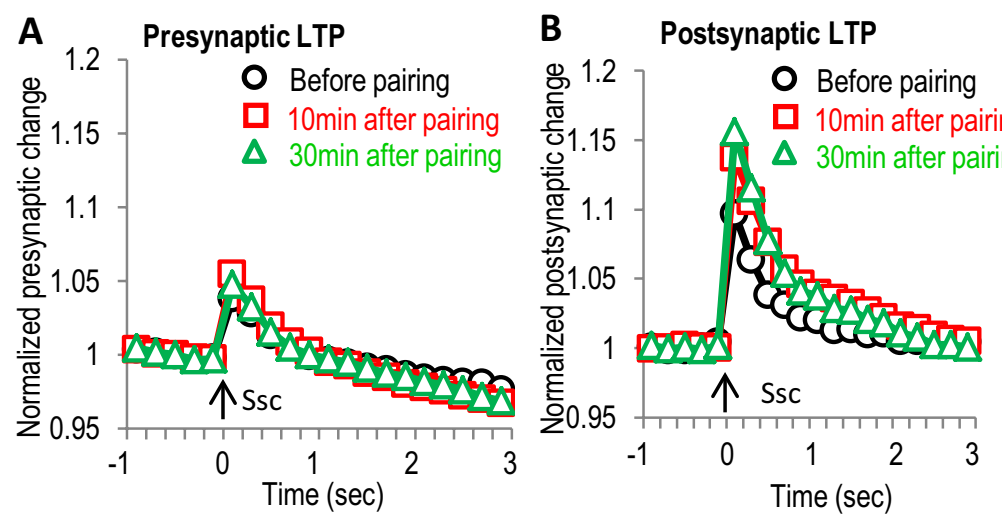

D
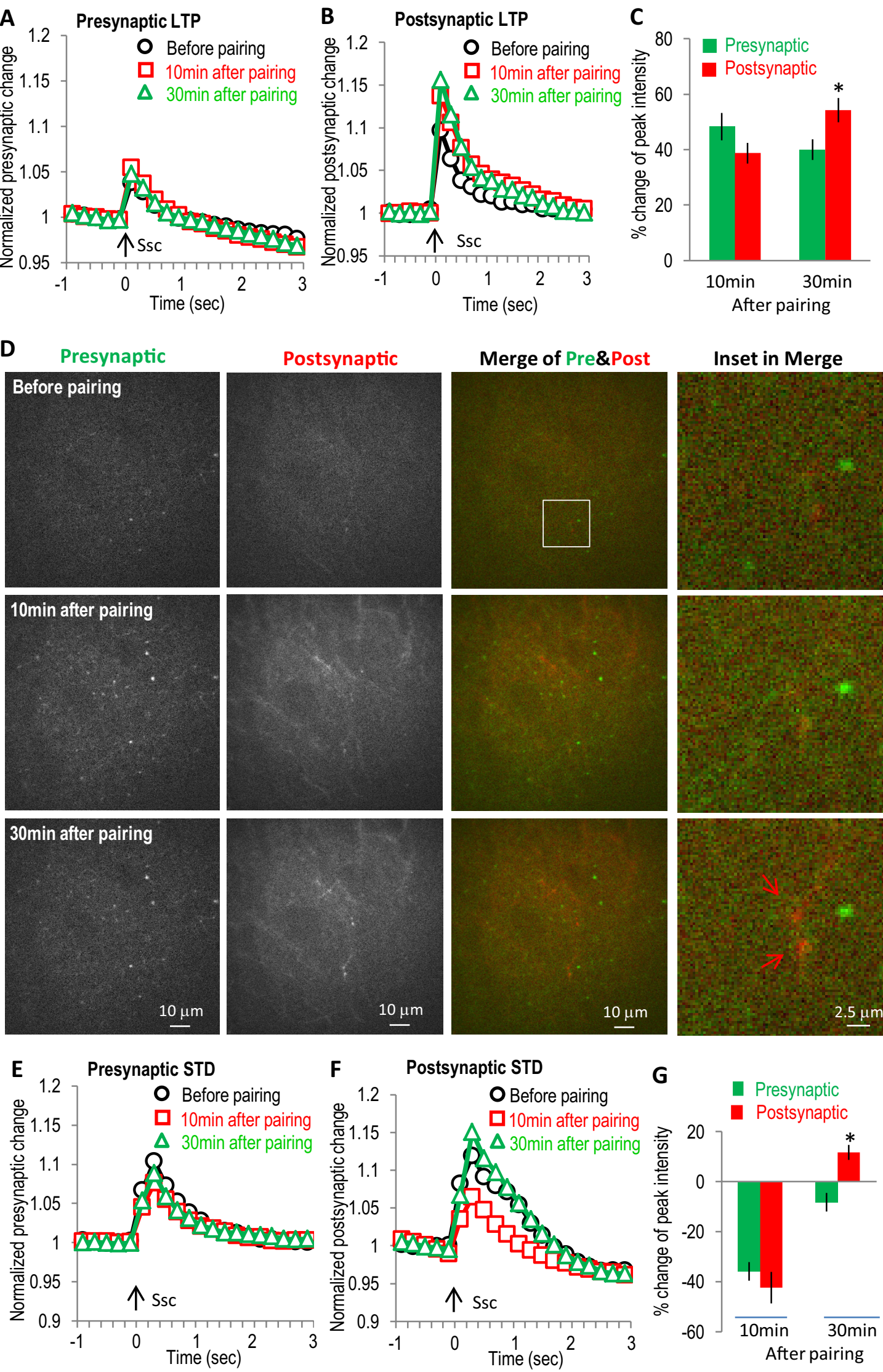

Figure 7. Differential presynaptic and postsynaptic changes in $\alpha 7$ nAChR-dependent LTP and STD revealed with dual-color GECl imaging. GECls (green GCaMP3 and red R-GEC01) were expressed separately in hippocampal CA3 and CA1 to monitor presynaptic and postsynaptic activities simultaneously for LTP $(\boldsymbol{A}-\boldsymbol{D})$ and STD $(\boldsymbol{E}-\boldsymbol{G})$. $\boldsymbol{A}$, Normalized presynaptic GCaMP3 responses showing smaller enhancement at $30 \mathrm{~min}$ than that at $10 \mathrm{~min}$. $\boldsymbol{B}$, Normalized postsynaptic R-GEC01 responses showing stronger enhancement at $30 \mathrm{~min}$ than that at $10 \mathrm{~min}$. $\boldsymbol{C}$, Bar graph showing the differential changes of the later stage (at $30 \mathrm{~min}$ ) between presynaptic and postsynaptic activities during the LTP. D, Images of presynaptic and postsynaptic (Figure legend continues.) 
changes with GECIs to investigate their individual contributions to the induction and expression of the $\alpha 7 \mathrm{nAChR}$-dependent LTP and STD. To do this, we used a coculture system whereby a slice from the septum was cultured next to a slice with hippocampus; in these cocultures, we confirmed that all three forms of plasticity that we had observed in acute slices was preserved. Then we expressed the GECIs into either the postsynaptic CA1 neurons or presynaptic CA3 neurons and monitored in the CA1 stratum radiatum region for postsynaptic (dendrites of CA1 neurons) and presynaptic (axons of CA3 neurons) activities, respectively. We observed a prolonged enhancement $(>30 \mathrm{~min}$ ) of the SC-induced calcium responses both postsynaptically and presynaptically in the $\alpha 7$ nAChR-dependent LTP. During the $\alpha 7 \mathrm{nAChR}$-dependent STD there was a short-term depression of the calcium responses both presynaptically and postsynaptically. Dual-color calcium imaging revealed a differential time course and pattern of postsynaptic versus presynaptic modulation during both LTP and STD (especially during the later stages), suggesting the existence of independent postsynaptic modulatory mechanisms. A similar time course between the presynaptic and postsynaptic changes would otherwise suggest that the postsynaptic change was merely the result of the presynaptic change.

To determine whether presynaptic or postsynaptic $\alpha 7$ receptors (or both) might be required for either LTP or STD, we expressed $\alpha 7$ receptors to either presynaptic or postsynaptic sites in slices from $\alpha 7$ nAChR knock-out (KO) mice. We found that the $\alpha 7$ receptors were required both presynaptically and postsynaptically to restore either LTP or STD in the $\alpha 7 \mathrm{nAChR} \mathrm{KO} \mathrm{slices.}$ This is consistent with the idea that there are independent postsynaptic and presynaptic modulations during the plasticity. Taken together, these results demonstrate that modulation at either presynaptic or postsynaptic sites alone is insufficient. Instead, coordinated modulation at both sites is required to induce either the LTP or the STD. Therefore, it appears that cholinergic inputs, through $\alpha 7 \mathrm{nAChRs}$ at both presynaptic and postsynaptic sites, can thus coordinate presynaptic and postsynaptic activities to induce timing-dependent synaptic plasticity.

Neuromodulator systems have been heavily implicated in higher brain functions and the induction of synaptic plasticity (Jerusalinsky et al., 1997; Bailey et al., 2000; Power et al., 2003; Dani and Bertrand, 2007; Kenney and Gould, 2008; Reis et al., 2009). The $\alpha 7 \mathrm{nAChRs}$, as well as other neuromodulator receptors, are known to be expressed at both the presynaptic and postsynaptic sites of glutamatergic and GABAergic terminals and can modulate synaptic excitability via presynaptic or postsynaptic mechanisms (Levey et al., 1991; Fabian-Fine et al., 2001; Sher et al., 2004; Volpicelli and Levey, 2004; Dani and Bertrand, 2007; Drever et al., 2011). The nAChR-dependent modulation of synaptic plasticity has been widely observed in the hippocampus and is thought to involve either presynaptic or postsynaptic mechanisms. For example, activation of nAChRs (both $\alpha 7$ and non- $\alpha 7$ receptors) in the CA1, dentate regions, and entorhinal cortex

\section{$\leftarrow$}

(Figure legend continued.) SC stimulation-induced calcium increases at different time points during the LTP. Left two panels show the active presynaptic and postsynaptic calcium responses to SC stimulation, respectively. The third panels are merged images of presynaptic and postsynaptic responses, with the right panels showing some potential spines. $\boldsymbol{E}$, Normalized presynaptic GCaMP3 responses showing the transient depression after the $S 0$ pathway pairing protocol. Arrow indicates stimulation of the SC pathway. $F$, Normalized postsynaptic R-GECO1 responses showing the transient depression after pairing the $S 0$ pathway. $\mathbf{G}$, Bar graph showing the differential recovery between presynaptic and postsynaptic activities during the STD. Ssc, Stimulation of the SC. enhances synaptic plasticity (Fujii et al., 1999; Mann and Greenfield, 2003; Tu et al., 2009; Ondrejcak et al., 2012). In addition, the activation of $\alpha 7 \mathrm{nAChRs}$ on hippocampal interneurons can block concurrent STP and LTP in pyramidal cells, whereas presynaptic nAChRs can enhance the release of glutamate and, thus, increase the probability of inducing LTP (Ji et al., 2001). However, the precise molecular mechanisms involving the induction and expression of synaptic plasticity and the role of presynaptic and postsynaptic changes remains unresolved and still much debated (Lisman, 2009; Glanzman, 2010). It is likely that in most cases, both presynaptic and postsynaptic components will be involved in the coordination and forming of synaptic plasticity. Here, we have provided direct evidence for presynaptic and postsynaptic modulation to cooperate in the induction of synaptic plasticity and, importantly, we show here that neuromodulator systems can serve as the mediator of such coordination.

The molecular mechanisms underlying the $\alpha 7 \mathrm{nAChR}$-mediated plasticity are not clear at this time, but may involve NMDA receptor activation and retrograde signaling, the two major molecular mechanisms proposed to connect the presynaptic and postsynaptic activities in inducing synaptic plasticity. The NMDA receptor, because of its depolarization-sensitive $\mathrm{Mg}^{2+}$ block, has been proposed as the coincidence detector for presynaptic and postsynaptic activities in both HFS-induced plasticity and STDP. We have previously shown that the $\alpha 7 \mathrm{nAChR}$-dependent LTP also involves NMDA receptor activation and prolonged postsynaptic intracellular calcium increase, suggesting that $\alpha 7 \mathrm{nAChR}$ activation may facilitate NMDA receptor activation. Retrograde signals (e.g., brain-derived neurotrophic factor or endocannabinoid) are released from the postsynaptic sites in an activity-dependent manner and affect presynaptic sites, thereby inducing plasticity (under the correct conditions). Neuromodulators can also facilitate retrograde messenger release with reduced calcium requirement, or in some cases even without calcium increases or postsynaptic firing (Kim et al., 2002; Best and Regehr, 2008; Regehr et al., 2009). However, other networks of neurons (for example GABAergic interneurons) or non-neuronal cells (such as astrocytes or glia) could aid in communication between the presynaptic and postsynaptic sites that is responsible for the prolonged increase in calcium responses observed both presynaptically and postsynaptically. For example, non-neuronal cells in the brain also express a variety of neurotransmitter receptors and ion channels (including the $\alpha 7 \mathrm{nAChRs)}$ (Vélez-Fort et al., 2009; Shen and Yakel, 2012) and participate in synaptic signaling and plasticity in the brain (Araque et al., 2002; Agulhon et al., 2008; Fiacco et al., 2009).

Neuromodulator-dependent associative heterosynaptic plasticity has several advantages over Hebbian homosynaptic plasticity (Bailey et al., 2000). First, it incorporates extrinsic signals into local networks, and second it significantly prolongs the expression of LTP and with higher efficiency and specificity. Currently, NMDA receptor-dependent homosynaptic plasticity is primarily induced by controlled postsynaptic depolarization through HFS or current injection. Little is known about how endogenous neuronal firing patterns lead to synaptic plasticity. Our study here has provided direct evidence that the precisely timed modulatory transmitter release can induce glutamatergic synaptic plasticity through coordinating the presynaptic and postsynaptic activities. Here, we propose that precisely timed activation of cholinergic inputs through the $\alpha 7 \mathrm{nAChRs}$, as well as potentially other neuromodulator systems, can coordinate the presynaptic and postsynaptic activities to induce synaptic plasticity with high efficiency and temporal precision. 


\section{References}

Agulhon C, Petravicz J, McMullen AB, Sweger EJ, Minton SK, Taves SR, Casper KB, Fiacco TA, McCarthy KD (2008) What is the role of astrocyte calcium in neurophysiology? Neuron 59:932-946.

Araque A, Martín ED, Perea G, Arellano JI, Buño W (2002) Synaptically released acetylcholine evokes $\mathrm{Ca}^{2+}$ elevations in astrocytes in hippocampal slices. J Neurosci 22:2443-2450.

Bailey CH, Giustetto M, Huang YY, Hawkins RD, Kandel ER (2000) Is heterosynaptic modulation essential for stabilizing Hebbian plasticity and memory? Nat Rev Neurosci 1:11-20.

Bastrikova N, Gardner GA, Reece JM, Jeromin A, Dudek SM (2008) Synapse elimination accompanies functional plasticity in hippocampal neurons. Proc Natl Acad Sci U S A 105:3123-3127.

Bell CC, Han VZ, Sugawara Y, Grant K (1997) Synaptic plasticity in a cerebellum-like structure depends on temporal order. Nature 387: $278-281$.

Best AR, Regehr WG (2008) Serotonin evokes endocannabinoid release and retrogradely suppresses excitatory synapses. J Neurosci 28:6508-6515.

Bliss TVP, Collingridge GLA (1993) Synaptic model of memory: long-term potentiation in the hippocampus. Nature 361:31-39.

Dan Y, Poo MM (2006) Spike timing-dependent plasticity: from synapse to perception. Physiol Rev 86:1033-1048.

Dani JA, Bertrand D (2007) Nicotinic acetylcholine receptors and nicotinic cholinergic mechanisms of the central nervous system. Annu Rev Pharmacol Toxicol 47:699-729.

Drever BD, Riedel G, Platt B (2011) The cholinergic system and hippocampal plasticity. Behav Brain Res 221:505-514.

Egger V, Feldmeyer D, Sakmann B (1999) Coincidence detection and changes of synaptic efficacy in spiny stellate neurons in rat barrel cortex. Nat Neurosci 2:1098-1105.

Fabian-Fine R, Skehel P, Errington ML, Davies HA, Sher E, Stewart MG, Fine A (2001) Ultrastructural distribution of the $\alpha 7$ nicotinic acetylcholine receptor subunit in rat hippocampus. J Neurosci 21:7993-8003.

Fayuk D, Yakel JL (2004) Regulation of nicotinic acetylcholine receptor channel function by acetylcholinesterase inhibitors in rat hippocampal CA1 interneurons. Mol Pharmacol 66:658-666.

Feldman DE (2000) Timing-based LTP and LTD at vertical inputs to layer II/III pyramidal cells in rat barrel cortex. Neuron 27: 45-56.

Fiacco TA, Agulhon C, McCarthy KD (2009) Sorting out astrocyte physiology from pharmacology. Annu Rev Pharmacol Toxicol 49:151-174.

Fischer Y, Gähwiler BH, Thompson SM (1999) Activation of intrinsic hippocampal theta oscillations by acetylcholine in rat septohippocampal cocultures. J Physiol. 519:405-413.

Fujii S, Ji Z, Morita N, Sumikawa K (1999) Acute and chronic nicotine exposure differentially facilitate the induction of LTP. Brain Res 846:137-143.

Gähwiler BH, Brown DA (1985) Functional innervation of cultured hippocampal neurones by cholinergic afferents from co-cultured septal explants. Nature 313:577-579.

Gähwiler BH, Hefti F (1984) Guidance of acetylcholinesterase-containing fibres by target tissue in co-cultured brain slices. Neuroscience 13:681-689.

Ge S, Dani JA (2005) Nicotinic acetylcholine receptors at glutamate synapses facilitate long-term depression or potentiation. J Neurosci 25:6084-6091.

Glanzman DL (2010) Common mechanisms of synaptic plasticity in vertebrates and invertebrates. Curr Biol 20:R31-R36.

Gu Z, Yakel JL (2011) Timing-dependent septal cholinergic induction of dynamic hippocampal synaptic plasticity. Neuron 71:155-165.

Jerusalinsky D, Kornisiuk E, Izquierdo I (1997) Cholinergic neurotransmission and synaptic plasticity concerning memory processing. Neurochem Res 22:507-515.

Ji D, Lape R, Dani JA (2001) Timing and location of nicotinic activity enhances or depresses hippocampal synaptic plasticity. Neuron 31:131-141.

Kenney JW, Gould TJ (2008) Modulation of hippocampus-dependent learning and synaptic plasticity by nicotine. Mol Neurobiol 38:101-121.

Kim J, Isokawa M, Ledent C, Alger BE (2002) Activation of muscarinic acetylcholine receptors enhances the release of endogenous cannabinoids in the hippocampus. J Neurosci 22:10182-10191.

Levey AI, Kitt CA, Simonds WF, Price DL, Brann MR (1991) Identification and localization of muscarinic acetylcholine receptor proteins in brain with subtype-specific antibodies J Neurosci 11:3218-3226.
Lisman JE (2009) The pre/post LTP debate. Neuron 63:281-284.

Magee JC, Johnston D (1997) synaptically controlled, associative signal for Hebbian plasticity in hippocampal neurons. Science 275:209-213.

Mann EO, Greenfield SA (2003) Novel modulatory mechanisms revealed by the sustained application of nicotine in the guinea-pig hippocampus in vitro. J Physiol 551:539-550.

Markram H, Lübke J, Frotscher M, Sakmann B (1997) Regulation of synaptic efficacy by coincidence of postsynaptic APs and EPSPs. Science 275:213-215.

Martin SJ, Grimwood PD, Morris RG (2000) Synaptic plasticity and memory: an evaluation of the hypothesis. Annu Rev Neurosci 23:649-711.

Neves G, Cooke SF, Bliss TV (2008) Synaptic plasticity, memory and the hippocampus: a neural network approach to causality. Nat Rev Neurosci 9:65-75.

Ondrejcak T, Wang Q, Kew JN, Virley DJ, Upton N, Anwyl R, Rowan M) (2012) Activation of $\alpha 7$ nicotinic acetylcholine receptors persistently enhances hippocampal synaptic transmission and prevents $A \beta$-mediated inhibition of LTP in the rat hippocampus. Eur J Pharmacol 677:63-70.

Power AE, Vazdarjanova A, McGaugh JL (2003) Muscarinic cholinergic influences in memory consolidation. Neurobiol Learn Mem 80:178-193.

Regehr WG, Carey MR, Best AR (2009) Activity-dependent regulation of synapses by retrograde messengers. Neuron 63:154-170.

Reis HJ, Guatimosim C, Paquet M, Santos M, Ribeiro FM, Kummer A, Schenatto G, Salgado JV, Vieira LB, Teixeira AL, Palotás A (2009) Neurotransmitters in the central nervous system and their implication in learning and memory processes. Curr Med Chem 16:796-840.

Rimvall K, Keller F, Waser PG (1985) Development of cholinergic projections in organotypic cultures of rat septum, hippocampus and cerebellum. Brain Res 351:267-278.

Séguéla P, Wadiche J, Dineley-Miller K, Dani JA, Patrick JW (1993) Molecular cloning, functional properties, and distribution of rat brain $\alpha_{7}$ : a nicotinic cation channel highly permeable to calcium. J Neurosci 13:596-604.

Shen JX, Yakel JL (2012) Functional $\alpha 7$ nicotinic ACh receptors on astrocytes in rat hippocampal CA1 slices. J Mol Neurosci. Advance online publication. Retrieved February 21, 2012. doi: 10.1007/s12031-012-9719-3.

Sher E, Chen Y, Sharples TJ, Broad LM, Benedetti G, Zwart R, McPhie GI, Pearson KH, Baldwinson T, De Filippi G (2004) Physiological roles of neuronal nicotinic receptor subtypes: new insights on the nicotinic modulation of neurotransmitter release, synaptic transmission and plasticity. Curr Top Med Chem 4:283-297.

Stoppini L, Buchs PA, Muller D (1991) A simple method for organotypic cultures of nervous tissue. J Neurosci Methods 37:173-182.

Tian L, Hires SA, Mao T, Huber D, Chiappe ME, Chalasani SH, Petreanu L, Akerboom J, McKinney SA, Schreiter ER, Bargmann CI, Jayaraman V, Svoboda K, Looger LL (2009) Imaging neural activity in worms, flies and mice with improved GCaMP calcium indicators. Nat Methods 6:875-881.

Tian L, Akerboom J, Schreiter ER, Looger LL (2012) Neural activity imaging with genetically encoded calcium indicators. Prog Brain Res 196:79-94.

Tsai HC, Zhang F, Adamantidis A, Stuber GD, Bonci A, de Lecea L, Deisseroth K (2009) Phasic firing in dopaminergic neurons is sufficient for behavioral conditioning. Science 324:1080-1084.

Tu B, Gu Z, Shen JX, Lamb PW, Yakel JL (2009) Characterization of a nicotine-sensitive neuronal population in rat entorhinal cortex. J Neurosci 29:10436-10448.

Vélez-Fort M, Audinat E, Angulo MC (2009) Functional alpha 7-containing nicotinic receptors of NG2-expressing cells in the hippocampus. Glia 57:1104-1114.

Volpicelli LA, Levey AI (2004) Muscarinic acetylcholine receptor subtypes in cerebral cortex and hippocampus. Prog Brain Res 145:59-66.

Witten IB, Lin SC, Brodsky M, Prakash R, Diester I, Anikeeva P, Gradinaru V, Ramakrishnan C, Deisseroth K (2010) Cholinergic interneurons control local circuit activity and cocaine conditioning. Science 330: 1677-1681.

Zhang LI, Tao HW, Holt CE, Harris WA, Poo M (1998) A critical window for cooperation and competition among developing retinotectal synapses. Nature 395:37-44.

Zhao Y, Araki S, Wu J, Teramoto T, Chang YF, Nakano M, Abdelfattah AS, Fujiwara M, Ishihara T, Nagai T, Campbell RE (2011) An expanded palette of genetically encoded $\mathrm{Ca}^{2+}$ indicators. Science 333:1888-1891. 